RESEARCH ARTICLE | Sensory Processing

\title{
Mechanical coupling through the skin affects whisker movements and tactile information encoding
}

\author{
(D) Valerie Ego-Stengel, Aamir Abbasi, Margot Larroche, Henri Lassagne, Yves Boubenec, and \\ Daniel E. Shulz \\ Department of Integrative and Computational Neuroscience, Paris-Saclay Institute of Neuroscience (NeuroPSI), UMR9197 \\ CNRS, University Paris-Sud, Gif-sur-Yvette, France
}

Submitted 21 December 2018; accepted in final form 6 August 2019

Ego-Stengel V, Abbasi A, Larroche M, Lassagne H, Boubenec Y, Shulz DE. Mechanical coupling through the skin affects whisker movements and tactile information encoding. J Neurophysiol 122: 1606-1622, 2019. First published August 14, 2019; doi:10.1152/ jn.00863.2018.- Rats use their whiskers to extract sensory information from their environment. While exploring, they analyze peripheral stimuli distributed over several whiskers. Previous studies have reported cross-whisker integration of information at several levels of the neuronal pathways from whisker follicles to the somatosensory cortex. In the present study, we investigated the possible coupling between whiskers at a preneuronal level, transmitted by the skin and muscles between follicles. First, we quantified the movement induced on one whisker by deflecting another whisker. Our results show significant mechanical coupling, predominantly when a given whisker's caudal neighbor in the same row is deflected. The magnitude of the effect was correlated with the diameter of the deflected whisker. In addition to changes in whisker angle, we observed curvature changes when the whisker shaft was constrained distally from the base. Second, we found that trigeminal ganglion neurons innervating a given whisker follicle fire action potentials in response to highmagnitude deflections of an adjacent whisker. This functional coupling also shows a bias toward the caudal neighbor located in the same row. Finally, we designed a two-whisker biomechanical model to investigate transmission of forces across follicles. Analysis of the whisker-follicle contact forces suggests that activation of mechanoreceptors in the ring sinus region could account for our electrophysiological results. The model can fully explain the observed caudal bias by the gradient in whisker diameter, with possible contribution of the intrinsic muscles connecting follicles. Overall, our study demonstrates the functional relevance of mechanical coupling on early information processing in the whisker system.

NEW \& NOTEWORTHY Rodents explore their environment actively by touching objects with their whiskers. A major challenge is to understand how sensory inputs from different whiskers are merged together to form a coherent tactile percept. We demonstrate that external sensory events on one whisker can influence the position of another whisker and, importantly, that they can trigger the activity of mechanoreceptors at its base. This cross-whisker interaction occurs pre-neuronally, through mechanical transmission of forces in the skin.

biomechanics; electrophysiology; tactile processing; whisker system

Address for reprint requests and other correspondence: V. Ego-Stengel, ICN, Neuro-PSI, CNRS, Bldg. 32/33, 1 avenue de la Terrasse, 91190 Gif-surYvette, France (e-mail: valerie.stengel@unic.cnrs-gif.fr).

\section{INTRODUCTION}

Rodents navigate and perform challenging tactile discriminations by touching surfaces and objects with their whiskers. The whisker system of the rat consists of $\sim 30$ whiskers on each side of the snout, which are arranged in a gridlike pattern. This striking discrete peripheral pattern is matched by the anatomical organization of upstream neuronal circuits in gridlike arrays of distinct substructures, called barrelettes, barreloids, and barrels in the brain stem, thalamus, and cortex, respectively (Ma and Woolsey 1984; Van Der Loos 1976; Woolsey and Van der Loos 1970). As a first-order description, sensory information travels in parallel pathways or "labelled lines" from each whisker to each cortical barrel (Deschênes and Urbain 2009).

However, in some of the first recordings in barrel cortex, neurons were already shown to exhibit responses to the individual deflection of several whiskers, demonstrating anatomical and functional convergence (Axelrad et al. 1976; Simons 1978). From a behavioral point of view, the analysis of information coming from several whiskers is indeed important for the animal to perform subtle discrimination tasks (Carvell and Simons 1995; Knutsen et al. 2006; Krupa et al. 2001). In fact, the complex patterns of whisker movements and contact characteristics are only beginning to be described in detail (Grant et al. 2009; Hobbs et al. 2016; Sherman et al. 2017). A major challenge will be to understand how multiple whisker inputs are merged together to form a coherent tactile percept. Despite an already large number of studies aimed at deciphering the mechanisms of multiwhisker integration, the anatomical and functional circuitry responsible for properties of cortical and thalamic receptive fields remains poorly understood.

At the most peripheral level, encoding of tactile stimuli is performed by several classes of mechanoreceptors located in the follicles at the base of the whiskers (Ebara et al. 2002, 2017). Approximately 150-200 first-order neurons from the trigeminal ganglion (TG) innervate each follicle (Vincent 1913) in an exclusive manner, that is, one TG neuron innervating only one follicle. This was originally inferred from functional studies, all of which reported that TG receptive fields contain a single whisker (Dykes 1975; Gibson and Welker 1983; Gottschaldt et al. 1973; Zucker and Welker 1969). It has been finally confirmed by anatomical means very recently (Tonomura et al. 2015). 
Upstream, cross-whisker connections have been found among brain stem nuclei (Jacquin et al. 1990; Voisin et al. 2002) and in the thalamo-cortico-thalamic loop (Arnold et al. 2001; Lavallée and Deschênes 2004). They are especially numerous intracortically (Bernardo et al. 1990; Narayanan et al. 2015), where they constitute a potential substrate for multiple forms of multiwhisker sensory integration (reviewed in Estebanez et al. 2018).

Nonetheless, there remains another possibility for early generation of cross-whisker signals, namely, that external contact forces on one whisker could lead to activation of mechanoreceptors in a neighbor follicle. Indeed, in the whisker system, sensory contacts occur on the shaft of the whisker, up to several centimeters away from the receptors in the follicle. Preneuronal treatment by the whisker itself transforms the dynamics of contact into a time course of forces at the base of the whisker (Bagdasarian et al. 2013; Boubenec et al. 2012, 2014; Quist and Hartmann 2012). How these forces then translate into mechanoreceptor activation has just started to be studied (Whiteley et al. 2015). Interestingly, the possibility of cross-whisker mechanical coupling has been suggested more than 30 years ago following the report of one TG neuron activated by a second whisker beyond its principal whisker (see Fig. 4 in Simons 1985). This study suggested the existence of "mechanical spread of the stimulus energy through the mystacial pad." Indeed, follicles are embedded in a complex mesh composed of skin, conjunctive tissue, and several muscles (Dörfl 1982; Haidarliu et al. 2010). Extrinsic muscles both for retraction (nasolabialis and maxillolabialis muscles) and protraction (nasalis muscle) run superficially, associated closely with the corium in the skin. Intrinsic muscles connect the top of each follicle with the deep part of its rostral neighbor. Interactions between follicles may be transmitted through the superficial layer of skin and/or via these different muscles.

We have developed two experimental approaches to study cross-whisker interactions. First, we have imaged individual whiskers using high-resolution videography of the snout of anesthetized rats while deflecting whiskers with high precision (Jacob et al. 2010). We quantified the deformation of a nondeflected whisker while another whisker was moved in terms of displacement, angle, and curvature. Second, we performed electrophysiological recordings of individual TG neurons. We investigated whether mechanical coupling can be sufficient to induce spikes in trigeminal neurons without stimulating their principal whisker. We integrate our results in a two-whisker biomechanical model bridging the gap between the external profile of the whisker on the one hand and the internal distribution of forces on the other hand, ultimately responsible for mechanoreceptor activation.

\section{MATERIALS AND METHODS}

\section{Animal Preparation}

All experiments were performed in conformity with French (Decree 2013-118, Ethics Committee project no. 3249-2015060516116339) and European (2010/63/EU) legislation on animal experimentation. Thirteen male Wistar rats (weight 250-300 g) were used in this study. Animals were housed in the NeuroPSI animal facility on a 12:12-h light-dark schedule, with two to four animals per cage. The animals were handled regularly and fed ad libitum. The experiment was always conducted during the light phase of the cycle. A group of seven rats was used for videography experiments, and another group of six rats was used for electrophysiology experiments. Atropine methyl nitrate $(0.3 \mathrm{mg} / \mathrm{kg}$ im) was injected to reduce secretions in the respiratory path. Rats were anesthetized with urethane $(1.5 \mathrm{~g} / \mathrm{kg} \mathrm{ip})$. The level of anesthesia was monitored by observing the absence of eye blink reflex, the lack of response to hind paw pinch, and the absence of spontaneous whisker movements. Supplementary doses of urethane $(0.15 \mathrm{~g} / \mathrm{kg}$ ip) were administered whenever necessary throughout the experiment to maintain an adequate level of anesthesia. Body temperature was maintained at $37^{\circ} \mathrm{C}$ by a regulated heating pad. The animal was placed in a stereotaxic frame. The snout was held by a modified head holder (Haidarliu 1996) allowing free access to the right whisker pad. A local anesthetic (lidocaine 1\%) was injected subcutaneously, and the skin on top of the skull was resected. After the conjunctive tissues were cleaned, the skull was cemented to a metal bar fixed rigidly to the frame. This allowed us to remove the right ear bar and to position the multiwhisker stimulator near the right whisker pad.

\section{Whisker Stimulation}

We used a custom-made whisker stimulation matrix based on piezoelectric benders (Jacob et al. 2010) to deflect independently the 24 most caudal whiskers of the right whisker pad. Whiskers were trimmed to $10 \mathrm{~mm}$ in length to avoid unwanted deflections due to whisker tips accidentally touching neighboring stimulators. Whiskers were inserted $3 \mathrm{~mm}$ into small polypropylene tubes glued on each bender (Polytec-PI), thus stimulated at $7 \mathrm{~mm}$ from their base. Benders were driven with resistor-capacitor (RC)-filtered voltage pulses producing a trapezoidal deflection. Our standard parameters produced pulses of 10-ms ramp, 10-ms plateau, and 10-ms ramp back, with an amplitude of $1^{\circ}$ applied at $7 \mathrm{~mm}$ from the follicle, in either a rostral or a caudal direction. We checked that the movement always stayed within $\pm 10 \%$ of its expected value by laser measurement.

\section{Videography Experiments}

High-speed, high-resolution video recording. A high-speed camera (Photron Fastcam SA3/105mm f-2.8 DG Macro Sigma) was mounted vertically above the animal to record the whisker movements at a $1-\mathrm{kHz}$ frame rate. The camera was triggered by a TTL sent by the whisker stimulator. Whiskers were illuminated from below using a backlight (SLLUB, Phlox; and PP520, Gardasoft). The camera was initially positioned to give a bird's-eye view of the $\mathrm{C} 2$ whisker and later translated above other whiskers. Given the geometrical constraints of the multiwhisker stimulator and the camera, we could only move the whiskers in a rostrocaudal direction and image them from the top. For calibration of the spatial scale of the camera field, we imaged a standard checkerboard sheet $(1 \mathrm{~mm} \times 1 \mathrm{~mm})$. Pixel resolution was checked for each series of movies and was in the range 16-20 $\mu \mathrm{m}$.

Whisker stimulation protocols. To study the effects of mechanical coupling across the whisker pad, we first imaged whisker C2 while deflecting each of the other 23 whiskers individually. For each deflected whisker, we performed four trials in the caudal direction and four trials in the rostral direction. Nonstimulated whiskers were let free in air (not inside the stimulator tips). The whole protocol was first applied while the imaged whisker C2 itself was free in air ("free" condition). The protocol was then repeated (in 4 of 7 experiments) while whisker $\mathrm{C} 2$ was constrained in its corresponding stimulator tip without movement ("constrained" condition).

In 3 of 7 experiments, we tested mechanical coupling effects on other whiskers in addition to whisker $\mathrm{C} 2$. Given the camera angle and snout geometry, we were able to image whiskers located in rows B to $\mathrm{D}$ and arcs 1 to 3 . Overall, we imaged 21 additional whiskers while deflecting either the immediately caudal or the immediately rostral adjacent whisker. Those tests were systematically done both in free and constrained conditions. For arc 1 whiskers, we chose to test their 
coupling on the caudal side with the straddler resulting in the smoothest alignment of follicles (B1-beta, C1-gamma, D1-delta), which correlates with the presence of an intrinsic muscle (Haidarliu et al. 2010).

Measurement of whisker parameters. At the end of the experiment, after all movies were acquired, we estimated the point of the whisker shaft corresponding to follicle entry for each imaged whisker. First, a wide-field snapshot of all whiskers and of the snout fur was taken. We then spread depilatory cream on the fur between the whiskers, let it set for 3-5 min, carefully removed the cream, and rinsed the pad. We took a second wide-field snapshot of the whisker pad without the fur, adjusting the lighting so that the entry of the whiskers in their follicles was clearly visible. Finally, whiskers were cut at the follicle entry and mounted on histology slides for measurement of their diameter and length under an optical microscope. For the D row, which was located below the pad outline and/or below other whiskers, the follicle entry position could often not be directly visualized. In those instances, it was estimated on the wide-field snapshot using the visible tip and the known length of the whisker, and taking into account the angle of the whisker relative to the horizontal plane of focus.

Data analysis. Camera recordings were analyzed using custom scripts in Python. Each movie contained 150 or 200 frames at $1 \mathrm{kHz}$, corresponding to one trial. Frames were typically $384 \times 512$ pixels. Their exact dimension was adjusted from one imaged whisker to the next depending on the viewing conditions. A series of eight trials (4 rostral, 4 caudal deflections) was analyzed for each pair of imaged and moved whiskers, and for each of the free and constrained conditions tested.

In all movies, the imaged whisker was approximately vertical on each frame, with the fur visible at the bottom (Fig. 1A). We defined a range of pixel lines in which the imaged whisker appeared clearly as a dark bar on a lighter background. For each line, the center of the whisker shaft was defined as the center of mass of the pixels encompassing the whole section of the whisker on that line (usually $\sim 15$ pixels), where each pixel is weighed by its intensity value compared with a given threshold. The threshold was adjusted independently for each imaged whisker. This yielded a raw profile of the whisker corresponding to the current frame (such as one colored line in Fig. 1). The computation was applied independently to each frame. We systematically checked whisker tracking for each movie by plotting several calculated raw profiles on top of their corresponding images. This allowed us to correct tracking problems due to unexpected changes (background element, global shift), mainly by changing the threshold or modifying the range of lines tracked.

Raw profiles of whiskers were never smooth, displaying many irregularities. Subpixel high-frequency spatial oscillations, dependent on the initial angle of the whisker relative to the vertical axis of the frames, could be ascribed to pixelization artifacts by the camera and were ignored in our analysis. We also encountered enlarged portions of a shaft, particles sticking on it, or bends. To focus on the changes over time irrespective of these singularities, we subtracted the profile calculated on the frame just before the start of the stimulation from all other profiles of the movie. We characterized the resulting deformation profiles, as well as the reference raw profile, by fitting each of them with a second-degree polynomial. This allowed us to extract three parameters quantifying the deformation: the displacement along the rostrocaudal axis, the change in whisker angle, and the change in curvature. These three parameters could be estimated at any point along the whisker shaft.

The tracked portion of the whisker was limited by the imaging constraints, in particular on the follicle side for which the view was obstructed by other whiskers and fur. We extrapolated the fits of the whisker profiles down to the estimated follicle entry. For population analysis, we filtered out trials for which the displacement near the tip or the change in angle near the follicle was outside of the range of the mean $\pm 1.5 \mathrm{SD}$, either in the baseline window 20 to $10 \mathrm{~ms}$ before the start of stimulation or in a second baseline window 10 to $20 \mathrm{~ms}$ after the end of stimulation. This eliminated $5-15 \%$ of trials on one given experiment, depending on the stability of the preparation.

To validate our method, in one animal we tracked the kinematic changes of whisker $\mathrm{C} 1$ while it was being deflected by its piezoelectric bender (Fig. 1, $A-D$ ). For each frame, a weighted average of pixel intensities across the whisker was performed line by line, in the region where the contrast of the whisker against the background was sufficiently good. The left side of Fig. $1 B$ shows the expanded resulting profiles of whisker $\mathrm{C} 1$ for 20 frames, corresponding to $20 \mathrm{~ms}$ during its deflection by the stimulator. The whisker tip was indeed deflected by the expected amount (114 $\mu \mathrm{m}$, i.e., $\sim 7$ pixels). Meanwhile, the whisker shaft showed a change in angle, which was largest toward the base of the whisker, as well as an increase in curvature that was best seen when looking at the deformation relative to rest (Fig. $1 C$, left). We fitted each profile independently by a second-order polynomial as described above. The fits (Fig. 1, $B$ and $C$ ) were extrapolated down to the estimated follicle entry point. Overall, the C1 imposed deformation appears as a rotation of the whisker around the follicle entry point with a change in curvature. We quantified several kinematic parameters at each point along the shaft: displacement in the rostrocaudal direction, angle relative to rest, and curvature. Figure $1 D$ displays the evolution of these parameters in time for the tip, middle, and follicle entry points. Note that residual ringing can be observed after ramp deflections, typical of piezoelectric stimulation (Jacob et al. 2010). Overall, these measures on the deflection of $\mathrm{C} 1$ confirm that our imaging method can measure subpixel deformations of a whisker at a $1-\mathrm{kHz}$ resolution.

We report median and interquartile range values. We performed nonparametric statistical tests because of low sample sizes. Individual tests are referred to in the main text.

\section{Electrophysiology Experiments}

Signal acquisition and spike sorting. In addition to the surgical steps for head fixation, a craniotomy was made on the skull overlaying the right TG $(1.8 \mathrm{~mm}$ posterior and $2.1 \mathrm{~mm}$ lateral from bregma; Schneider et al. 1981). A dam of dental acrylic was constructed around the craniotomy and filled with saline to prevent the brain from drying. Extracellular neural activity was recorded from a tungsten electrode (FHC; $2-10 \mathrm{M} \Omega$ at $1 \mathrm{kHz}$ ) that was vertically lowered $\sim 10$ $\mathrm{mm}$ down in the TG using an electronically controlled manipulator (Luigs and Neumann). Custom-made software (Elphy; G. Sadoc, UNIC, France) was used for spike time acquisition, whisker stimulation, and data processing. In a first series of experiments ( 5 cells in 2 rats), signals were amplified and filtered $(300-3,000 \mathrm{~Hz})$ by an acquisition card (CyberAmp) connected to a template-matching hardware spike sorter (Alpha-Omega). In a second series ( 9 cells in 4 rats), signals were amplified and filtered $(250-7,500 \mathrm{~Hz})$ using a different acquisition system (Blackrock Microsystems). Single units were isolated using the integrated online spike sorter. In all experiments, baseline signals had a standard deviation of 10-15 $\mu \mathrm{V}$. Single-unit spike waveforms had amplitudes of $100 \mu \mathrm{V}$ or more. Because cell density and cell firing are sparse in the TG, we typically recorded at most one or a few action potentials per stimulus separated by long periods of silence. Thus action potential waveforms were clearly separated from the noise. The shape of action potentials was closely monitored online to ensure that only isolated single units were recorded throughout the protocols. The recording was terminated if the quality of spike classification was lost. At the end of recording at a given site, the electrode was advanced by at least $100 \mu \mathrm{m}$ before the next recording site to avoid recording data from the same single units.

Whisker stimulation protocols. We first characterized the receptive field of each neuron by presenting pseudorandom sequences of 30100 individual deflections of the 24 whiskers in the rostral and caudal directions. Initially, we applied pulses of $0.93^{\circ}$ ( 2 experiments) or $1^{\circ}$ (4 experiments), corresponding to angular speeds of $93-100^{\circ}$ s. Once the receptive field of the neuron was established, we tested mechanical 


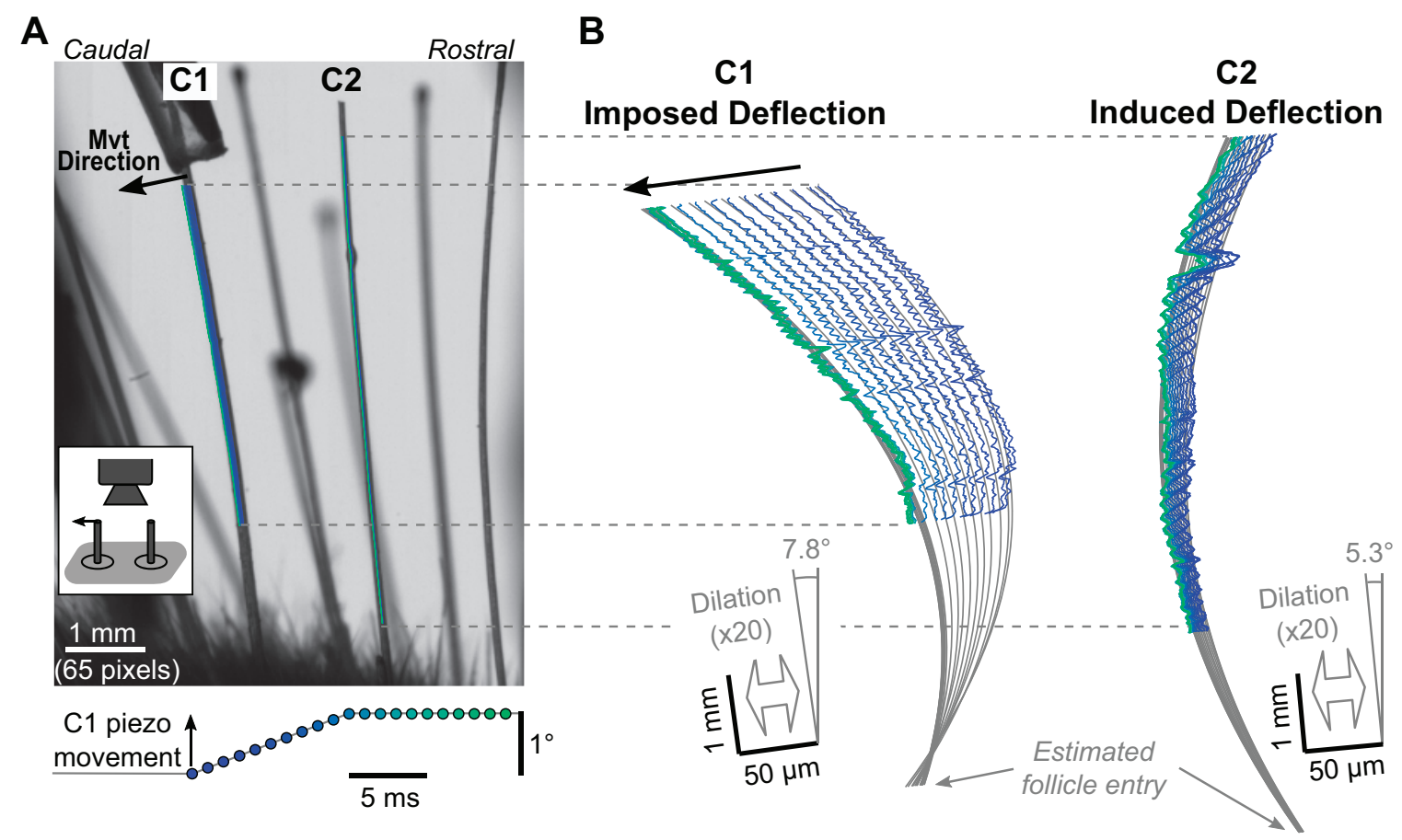

C

\section{C1 Deformation C2 Deformation}

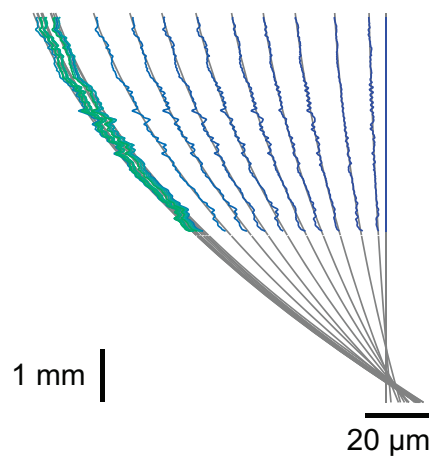

D

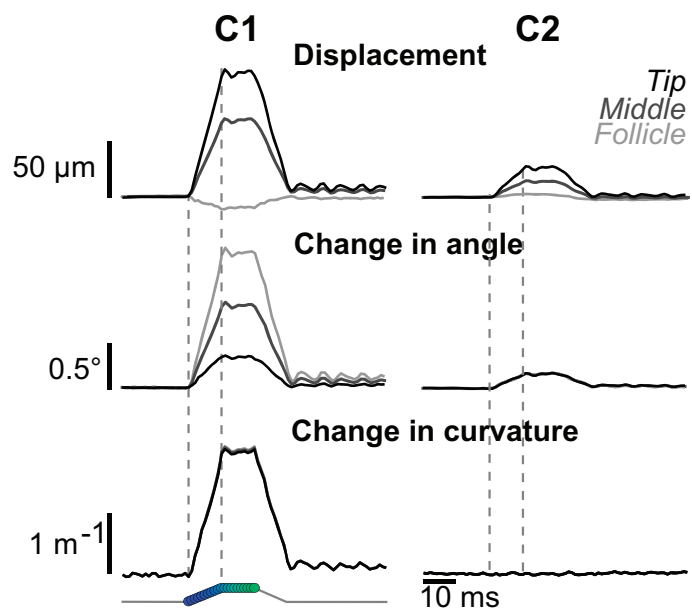

Fig. 1. An imposed $1^{\circ}$ deflection of whisker $\mathrm{C} 1$ induces a rotation of whisker $\mathrm{C} 2$ around its follicle entry. $A$ : raw image of the region of interest captured during study of the effect on whisker $\mathrm{C} 2$ of a ramp-plateau-ramp deflection of whisker $\mathrm{C} 1$. Time course of the $\mathrm{C} 1$ deflection is indicated below the image, along with the color code for 20 successive frames acquired at $1 \mathrm{kHz}$ during the up-ramp and plateau of the pulse. The $\mathrm{C} 1$ and $\mathrm{C} 2$ whisker profiles calculated from these 20 frames are superimposed on the image. $B$ : the same 20 raw profiles are displayed after a magnification factor (dilation) of gain 20 was applied in the direction orthogonal to the whisker. This anisotropic manipulation, solely used for display, was necessary to effectively observe the induced displacements. With these scales, the whisker profiles appear bumpy as a consequence of irregularities of the shaft and camera pixelization. Gray lines are second-order polynomial fits, extrapolated down to follicle entry estimates. $C$ : deformation calculated by subtracting the profile right before the start of the deflection and after smoothing with a time window of $5(\mathrm{C} 1)$ or 10 points $(\mathrm{C} 2)$ solely for visual display. Note horizontal scales are different for the 2 whiskers. Curvature changes could be reliably visualized on these plots, whereas they were difficult to see before the subtraction. $D$ : time course of displacement, change in angle, and change in curvature for the 2 whiskers at 3 different levels along the shaft (tip, middle, follicle). "Tip" indicates the point at which the stimulation contacts the whisker shaft. The induced deflection on $\mathrm{C} 2$ is a rigid rotation around the follicle entry, with no curvature change. Mvt, movement.

coupling across an increasing range of ramp speeds. We increased the deflection amplitude to $3^{\circ}$ or $4^{\circ}$, resulting in an increase in ramp speed to 300 or $400 \%$, respectively. We also reduced the ramp duration from $10 \mathrm{~ms}$ to 5 or $3 \mathrm{~ms}$, to increase the deflection speed up to $1,200^{\circ} / \mathrm{s}$. In a few cases, we approached further the stimulator tip along adjacent whiskers down to $\sim 3 \mathrm{~mm}$ from the follicle, which resulted in an increased speed of the deflection to $\sim 4,000 \%$ s. These different parameter modifications were tested until a coupling effect was observed from one adjacent whisker, at which point we stopped increasing ramp speed. Interestingly, all neurons for which we were able to test this range of increasing ramp speeds displayed mechanical coupling effects for at least one adjacent whisker. For other neurons, the quality of single-unit isolation was lost before high speeds could be tested.

Beyond a direct response of a primary afferent neuron to one of the adjacent whiskers, we tested whether a movement of an adjacent whisker could modify the response of the neuron to deflections of its principal whisker, through a subthreshold modulation. We first determined deflection parameters for the coupled adjacent whisker that elicited no response for either direction of movement. We then stimulated the principal whisker in its preferred direction with eight different pulses of increasing speed and fixed amplitude stimuli, 
obtaining a response curve as a function of speed. Once all parameters were determined, we studied the modulation of this curve by adding subthreshold deflections to the adjacent whisker in either direction. Trials with only the principal whisker deflected, or with deflections of both the principal whisker and the adjacent whisker in either rostral or caudal direction, were pseudorandomly interleaved. We could only complete this final protocol satisfactorily in one case (see Fig. 6).

Data analysis. Peristimulus time histograms were constructed by summing the activity of the neuron relative to the stimulus trigger with a 1-ms time bin. Spontaneous activity was null for 13 of 14 neurons and below $1 \mathrm{~Hz}$ for the remaining neuron.

\section{Biomechanical Model}

A finite-element model of two whiskers and follicles was built in SolidWorks Simulation. Geometrical parameters such as whisker diameter, follicle dimensions, and inter-whisker spacing are known to vary across the whisker pad. We used values obtained from the literature (Haidarliu et al. 2010; Kim et al. 2011) and complemented from our own measurements taken in the center of the whisker pad on and around $\mathrm{C} 2$. Note that the chosen geometry needed to be compatible with a mesh model, thus avoiding very small features and very high curvature surfaces. This forced us in particular to use a larger whisker diameter than typical values for the rat. Hence, each whisker was modeled by a rod of diameter $300 \mu \mathrm{m}$, i.e., two times thicker than the $\mathrm{C} 2$ whisker of our animals (149 $\mu \mathrm{m}, n=4$ rats; see also Belli et al. 2017). To investigate the impact of whisker diameter on the forces inside the follicle, we also tested diameters of 250 and $350 \mu \mathrm{m}$ (see RESULTS). The whisker rod was $10 \mathrm{~mm}$ long, modeling a cut whisker of which the tip would be manipulated. Each follicle was modeled by a cylinder of diameter $800 \mu \mathrm{m}$ and length $2.5 \mathrm{~mm}$ in which a whisker was inserted. Follicle centers were $2 \mathrm{~mm}$ apart. Follicles and whiskers were attached at their base to a fixed plate. A layer of skin was added in which the top of the follicles was embedded. This rectangular skin component had a thickness of $80 \mu \mathrm{m}$ and extended $750 \mu \mathrm{m}$ in each direction from the follicle borders. We did not attempt to model the extrinsic muscles running along the corium separately, but considered the skin sheet as including those muscles. For one set of simulations, we modeled the intrinsic muscle as a single rod connecting two rings, one around the caudal follicle just below the skin and one around the rostral follicle centered at a depth of two-thirds the total follicle depth. The ring was $200 \mu \mathrm{m}$ thick and $300 \mu \mathrm{m}$ high. The connecting rod had a diameter of $100 \mu \mathrm{m}$.

Follicles and skin, as well as the intrinsic muscle when simulations included one, were modeled by a material with mechanical parameters close to rubber with Young's modulus $=0.12 \mathrm{GPa}$ and Poisson's ratio $=0.49$. Whiskers were modeled by a material close to polyvinyl chloride with Young's modulus $=7.2 \mathrm{GPa}$, in agreement with measures present in the literature (Carl et al. 2012; Hartmann et al. 2003; Neimark et al. 2003), and Poisson's ratio $=0.38$.

The contact surfaces between components did not allow penetration. Follicles and skin, as well as the intrinsic muscle when present, were bonded, whereas follicles and whiskers could separate. The mesh size was $80 \mu \mathrm{m}$, which resulted in 51,567 elements and 83,978 nodes for our default geometrical parameters. Whisker deflection was modeled by a rotation of the whisker tip of $1^{\circ}$ around the whisker base center at the bottom of the follicle. The neighboring whisker distal tip could be either free or constrained (fixed). These boundary conditions on the deflected whisker and its neighbor imposed the whisker angles at the distal end, which was not the case in the experimental conditions. The distal whisker profiles could thus sometimes differ from the observed ones. This did not affect deformations near and inside the follicles, which were the focus of our study.

Simulations were run for both rostral and caudal directions of movement. Because results were always found to be symmetric, we chose to report the effect of deflecting one whisker toward the other. Thus the rostral whisker was deflected caudally, and the caudal whisker was deflected rostrally. The simulations all assumed a linear elastic behavior of the components and were restricted to small displacements.

\section{RESULTS}

\section{High-Speed Videography Reveals Whisker Movements Induced by Mechanical Coupling}

We investigated mechanical coupling between whiskers by imaging directly the whiskers on the snout of rats $(n=7$ animals) using a high-frame rate, high-resolution camera and a custom-built multiwhisker stimulator (Jacob et al. 2010).

First, we validated our tracking method by verifying that the movement of a whisker deflected by a piezoelectric bender could be imaged at $1 \mathrm{kHz}$ and quantified with adequate spatial resolution (Fig. 1, $A-D$, whisker C1; see MATERIALS AND METHODS). We then applied this tracking method to the neighboring whisker $\mathrm{C} 2$. As shown by the raw profiles and fits in Fig. $1 B$, whisker $\mathrm{C} 2$ also moved during the deflection of whisker $\mathrm{C} 1$, even though it was not directly deflected by the experimenter. Moving whisker $\mathrm{C} 1$ induced a displacement at the tip of C2 of $25 \mu \mathrm{m}$, representing $23 \%$ of the tip displacement imposed on $\mathrm{C} 1$. For both the deflected and the imaged whisker, there was little if any translation at the base of the whisker, as estimated by extrapolating the whisker profile down to the follicle entry point. The change in angle along whisker $\mathrm{C} 2$ reached $0.16^{\circ}$, that is, $11 \%$ of the imposed angle at the $\mathrm{C} 1$ follicle entry. There was no change in curvature of whisker $\mathrm{C} 2$ so that the overall deformation was well described by a change in angle (Fig. 1, $C$ and $D$, right). These results demonstrate that even for small movements, there can be a measurable mechanical coupling between two neighboring whiskers. Deflection of one whisker induced a rigid transformation of its rostral neighbor, more precisely, a rotation around the follicle entry point.

\section{Mechanical Coupling Is Strongest from a Caudal Whisker in the Same Row}

We explored the effect of deflecting one by one each whisker, always measuring the movement of the nondeflected central whisker C2. The time course of the angular rotation of $\mathrm{C} 2$ for all trials of one experiment is shown on Fig. 2A, separately for deflections in the caudal (left) and rostral (right) directions. For each given deflected whisker, the observed profile was highly repeatable from one trial to the next. In this example, we observed mechanical coupling for deflections of whiskers beta, B1, gamma, C1, and C3. The effect was similar for deflections in the caudal and rostral directions, showing mirroring profiles. We thus pooled induced effects across the two conditions for all subsequent population analysis and figures. Analysis from seven experiments confirmed a consistent gradient of the amplitude of induced movement of $\mathrm{C} 2$ during movement of other whiskers around it (Fig. 2B). As observed in Fig. 1, induced movements were rigid rotations along the estimated follicle entry, with no curvature change or translation at the base of the whisker. For further analysis, we thus focused on changes in whisker angles (middle matrix of Fig. $2 B$ ). The effect tended to decrease as the distance between $\mathrm{C} 2$ and the deflected whisker increased. However, distance was clearly not the only factor determining the amplitude of the me- 
A

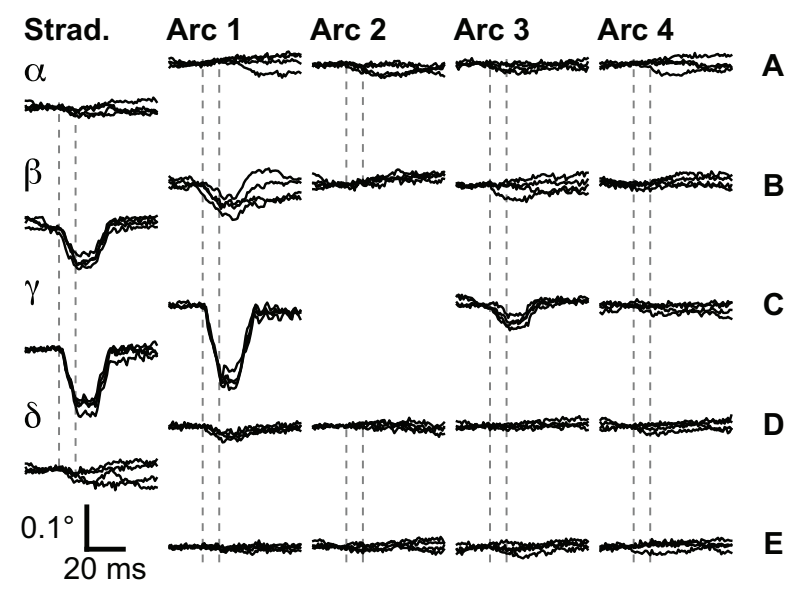

\section{Rostral Deflection}

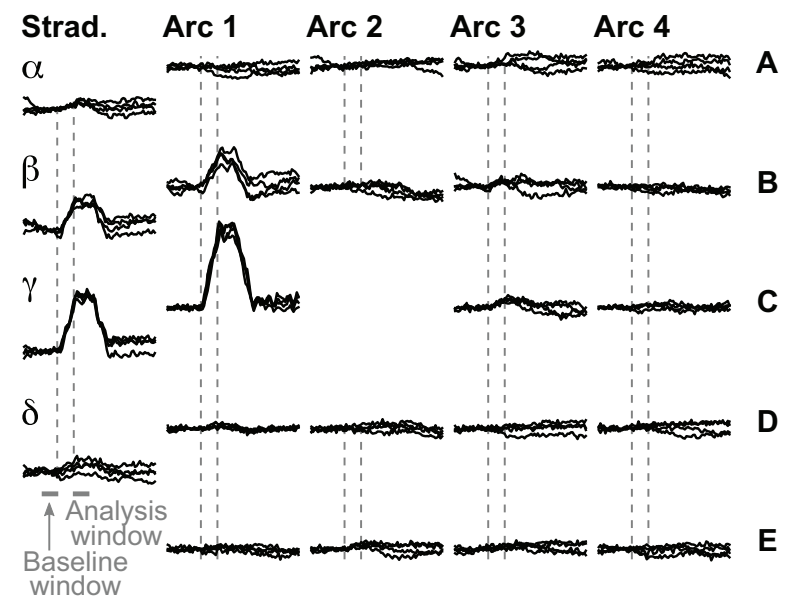

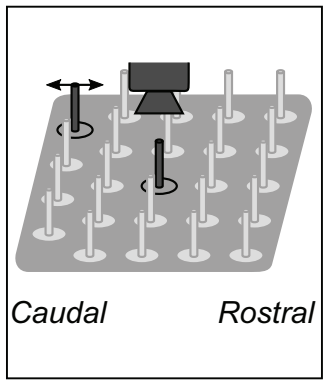
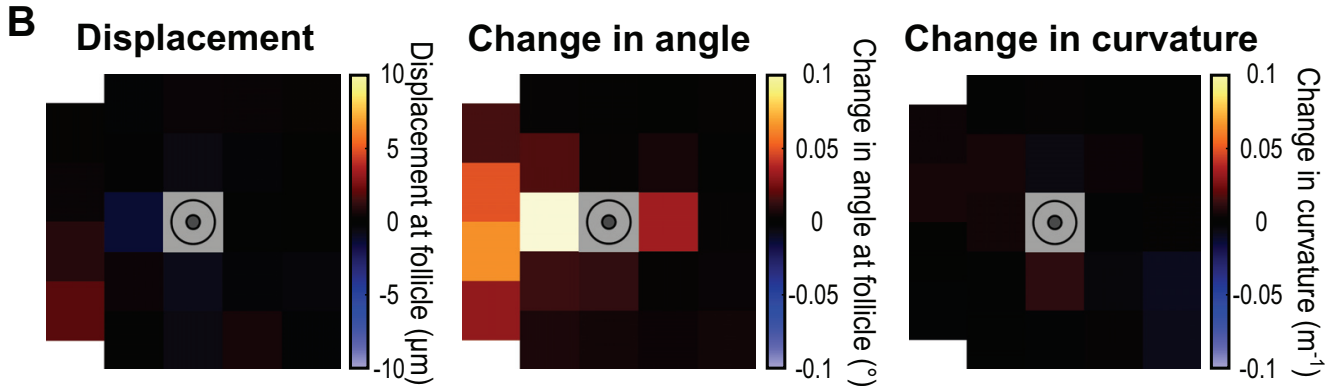

Fig. 2. Spatial map of mechanical coupling effects on whisker $\mathrm{C} 2$ from other whiskers on the pad. $A$ : time course of whisker angle at the follicle level while every other whisker on the pad is deflected in either a caudal or rostral direction. Traces are aligned around their mean calculated in the 10-ms window before the stimulus. Strad., straddlers arc; $A-E$ indicate rows; alpha, beta gamma, and delta indicate straddler whiskers between rows. $B$ : displacement, change in angle, and change in curvature at the follicle level quantified in the time window 10-20 ms after stimulation (plateau of pulse) and pooled over rostral and caudal directions (median values over 7 rats).

chanical coupling. We observed a strong asymmetry among whiskers, with whiskers caudal to C2 generating much larger induced movements than those located rostrally. Also, whiskers in the same row as C2 were more effective, suggesting an effect more potent along rows than along arcs.

\section{Strength of Mechanical Coupling Depends on Whisker Location}

Given that not all whisker pairs exhibited detectable mechanical coupling, we wondered which parameters govern the amplitude of the coupling effect. Figure $2 B$ suggests that distance between the whiskers is an important factor, as well as location in the same row. Also, it points to an asymmetry depending on whether the deflected whisker is located rostrally or caudally to the imaged whisker. However, these results were obtained by always imaging $\mathrm{C} 2$ so that the asymmetry could also be due to the identity of the whisker moved, and not to its rostral vs. caudal location relative to the imaged whisker.

We decided to investigate this question further by testing other combinations of whiskers distributed across the whisker pad. We focused on immediately neighboring pairs in one row, for which the effect was expected to be largest and for which the distance between the follicles was always around $2 \mathrm{~mm}$. The results of this data set are summarized in Fig. 3. First, we checked whether the asymmetry observed for whisker C2 in Fig. 2 held when results were analyzed from all whiskers imaged, located in rows B-D and arcs 1-3. Indeed, the coupling effect was consistently strongest when the whisker immediately caudal was deflected, compared with the rostral one (Fig. 3A; Wilcoxon signed-rank test, $P=1.2 \times 10^{-5}$ ). In this same data set, we could also ask whether for a particular combination of whiskers, the effect was similar whichever whisker was the deflected one. We found again that induced movements were larger when the caudal whisker was deflected, compared with the rostral whisker (Fig. 3B; Wilcoxon signed-rank test, $P=0.0023)$. Moreover, when we compared the impact of deflecting a fixed whisker on its two immediate neighbors, we found that the effect was strongest on the rostral whisker compared with the caudal one in each of the five cases where we could image on both sides (Fig. 3C; Wilcoxon signed-rank test, $P=0.043$ ). Together, these results point to a consistent underlying asymmetry such that mechanical coupling is strongest when the whisker inducing the movement of its neighbor is located caudally to it. Pooling results from all experiments, we looked at whether there was a systematic bias due to the location of the whiskers on the whisker pad (Fig. $3 D$ ). We observed strong variations in the coupling amplitude across the whisker pad, with larger effects in more caudal and ventral locations.

We reasoned that this spatial distribution must arise from a systematic gradient in one or several mechanical parameters across the whisker pad. Many such gradients have been re- 
ported, and all of them follow a set of consistent rules. In particular, from rostrodorsal to caudoventral locations, the whisker diameter increases sharply (Belli et al. 2017; Ibrahim and Wright 1975; Voges et al. 2012), along with a moderate increase in follicle size and distance between follicles (Haidarliu et al. 2010; also observed in our sample). Because the thickness of the deflected whisker directly governs its mechanical rigidity, it could have a strong impact on the surrounding skin, including neighboring follicles and whiskers. We thus examined the relation between the diameter of the whiskers, measured in four animals, and the amplitude of the coupling effect. Median diameters are indicated in Fig. $3 D$ and, as expected, covaried with the size of the coupling effect. Population

A

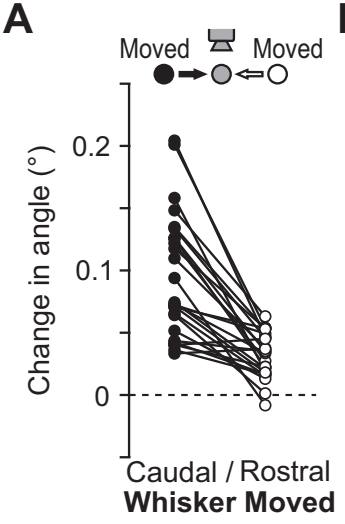

B

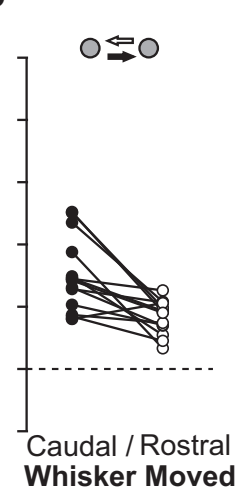

C

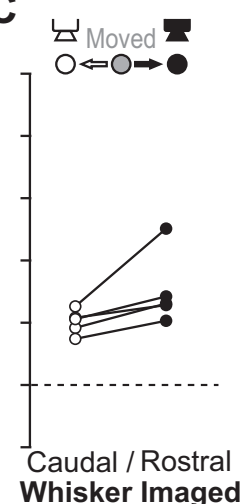

D

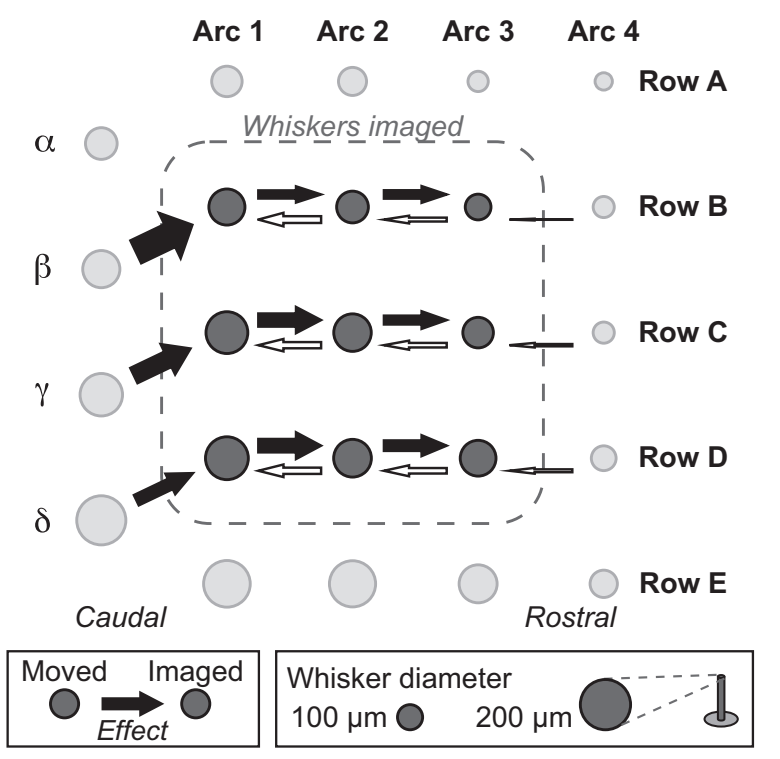

E

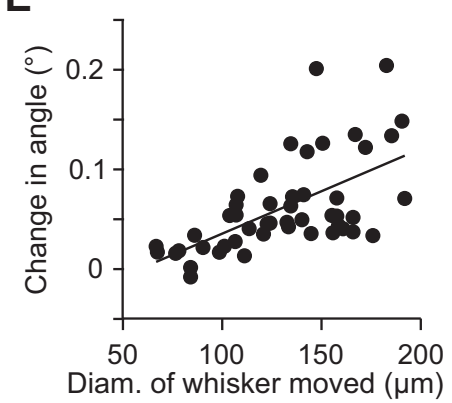

$\mathbf{F}$

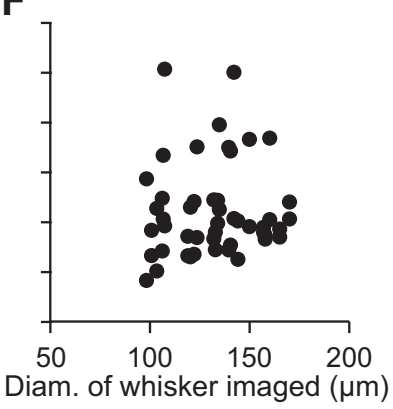

scatter plots confirmed a significant correlation between the observed coupling effect and the diameter of the moved whisker (Fig. 3E; Spearman's coefficient $\rho=0.63, P=1.27 \times 10^{-6}$ ) and less with the diameter of the imaged whisker (Fig. $3 F$; Spearman's coefficient $\rho=0.14, P=0.034)$. We conclude from these data that mechanical coupling is dependent on properties local to the deflected whisker, such as its diameter. Nonetheless, we acknowledge that beyond the gradient of diameter of the deflected whisker, other gradients of mechanical properties of the whiskers, follicles, or skin may contribute to the observed asymmetries. Because all these gradients are correlated, it is difficult to disentangle their relative contribution. This is best addressed by manipulation of individual features in a biomechanical model, as we report in Mechanical Model of Two Neighboring Whiskers and Follicles.

\section{Constraining the Whisker Tip Induces Curvature Changes}

In all these observations, the imaged whisker was unrestrained while other whiskers were deflected. The induced movement consisted in a rotation around the follicle entry with no detectable change in curvature or displacement of the follicle entry point (Figs. 1 and $2 B$ ). In previous studies modeling the whisker as a rigid anchored beam, changes in curvature have been shown to be proportional to the moment of rotational forces along the whisker (Quist and Hartmann 2012; Solomon and Hartmann 2006). Our results reported above are consistent with the fact that since the whisker shaft was not touching any external object, there were no forces along it, and thus no curvature changes. Mechanical conditions are different when the shaft of the whisker is maintained in a given position or manipulated by a stimulator. The whisker is then constrained both at the follicle level and by the external contact. Forces are generated along the whisker shaft, and the whisker bends. In those conditions, the curvature changes give an estimate of the forces generated along the whisker shaft down to the follicle entry, where mechanoreceptors are located.

Fig. 3. Mechanical coupling between neighbors follows a systematic gradient on the whisker pad. A: median change in angle for a given imaged whisker when its caudal neighbor (closed circles) or rostral neighbor (open circles) was moved ( $n=26$ whisker pairs). The change in angle was significantly higher when the caudal whisker was moved (Wilcoxon signed-rank test, $P=1.2 \times$ $10^{-5}$ ). In $A-F$, closed (or open) arrows indicate mechanical coupling due to deflection of a caudal (or rostral) whisker. $B$ : median change in angle for a given whisker combination when the caudal whisker was moved and the rostral whisker was imaged (closed circles) vs. when the rostral whisker was moved and the caudal whisker was imaged (open circles) ( $n=14$ whisker pairs). The change in angle was significantly higher when the caudal whisker was moved (Wilcoxon signed-rank test, $P=0.0023$ ). $C$ : median change in angle for a given deflected whisker when its caudal neighbor (open circles) or rostral neighbor (closed circles) was imaged ( $n=5$ whisker pairs). The change in angle was significantly higher when the rostral whisker was imaged (Wilcoxon signed-rank test, $P=0.043)$. $D$ : summary of the mechanical coupling effect pooled across experiments. The width of each arrow indicates the mean change in angle induced on one whisker (located at the end of the arrow) while a neighboring whisker (located at the start of the arrow) was deflected ( $n=52$ effects tested on 6 rats; $1-3$ data points per arrow, except 6 data points for C2 imaged). Circles indicate the median diameter of each whisker ( $n=4$ rats). $E$ : median change in angle as a function of the diameter of the whisker moved ( $n=48$ whisker pairs). There was a significant positive correlation between these variables (Spearman's coefficient $\left.\rho=0.63, P=1.3 \times 10^{-6}\right) . F$ : median change in angle as a function of the diameter of the whisker imaged ( $n=48$ whisker pairs). These variables were not significantly correlated (Spearman's coefficient $\rho=0.14, P=0.034)$. 
To investigate these forces during mechanical coupling, we repeated the measures of whisker deformation after introducing the imaged whisker into the standard plastic cylinder attached to our multiwhisker stimulator, in the rest position. In this constrained configuration, deflecting a whisker could still induce a measurable movement in a neighboring whisker (Fig. $4 A)$. As expected, the amplitude of the deformation and the change in angle were smaller than in the free condition (note the different horizontal scales for the profiles of Fig. 4A). However, we now observed a change in the whisker curvature. These results were confirmed in our population data set of videography recordings in which one whisker was imaged while its immediate neighbor in the same row was deflected, either free or constrained (Fig. 4, $B$ and $C, n=46$ whisker pairs). Specifically, changes in angle that were observed in the free condition were correlated with changes in angle of smaller amplitude in the constrained condition (Fig. 4B; Spearman's coefficient $\rho=-0.56, P=5.4 \times 10^{-5}$ ), as in the example of Fig. $4 A$. Additionally, in the constrained condition, changes in curvature were significantly correlated with changes in angle (Fig. 4C; Spearman's coefficient $\rho=0.47, P=0.001$ ).

These results emphasize that in the constrained condition, because of the added external force at the tip counteracting the natural movement of the whisker, rotational forces are generated along the imaged whisker down to its base. As a consequence, below follicle entry, the distribution of forces at the whisker-follicle contact surface is likely to be different in the constrained vs. the free condition, leading to possible differences of mechanoreceptor activation.

\section{Deflection of Adjacent Whiskers at High Amplitude Evoke Action Potentials in TG Neurons}

The whisker imaging experiments demonstrated that neighboring whiskers and their follicles are indeed distorted when a single whisker is deflected. Next, we wanted to assess whether mechanical coupling could directly elicit spiking activity in primary afferent axons. We recorded extracellularly from TG neurons while stimulating the ipsilateral whiskers in six anesthetized rats. All 24 whiskers were constrained in their respective stimulators throughout the experiment to minimize manipulation of the animal snout during the electrophysiological recordings. Because TG neurons each have a different threshold for evoked activity, we routinely tested several speeds and amplitudes of stimulation to determine both the whisker follicle innervated by each neuron and the stimulation threshold. In each case, we determined a relatively low level of stimulation at which we observed evoked spikes for the deflection of only 1 whisker out of 24 . This is in line with previous studies of TG receptive fields, reported to be monovibrissal (Gibson and Welker 1983; Zucker and Welker 1969). For example, the first neuron displayed in Fig. $5 A$ responded only to the deflection of whisker E1, and only in the caudal direction (top row of rasters and histograms). There was no spiking for the rostral direction of movement, or for either direction when the stimulation was applied on any of the other 23 whiskers (shown as an example for whisker delta). We conclude that E1 is the principal whisker of this neuron.

To estimate the impact of mechanical coupling on the firing of this neuron, we then stimulated all whiskers individually at a higher speed $(1,330 \%$ s vs. $800 \%$ s previously). Spikes were now reliably evoked following the deflection of the adjacent whisker delta in the rostral direction (Fig. 5A, second row of rasters and histograms). This additional response indicates sufficient mechanical coupling between follicles delta and E1 to induce spikes in the E1-innervating neuron when deflecting delta. The response disappeared entirely if we removed the E1 whisker from its stimulator, indicating that the constrained state of the principal whisker contributed to the mechanical
A
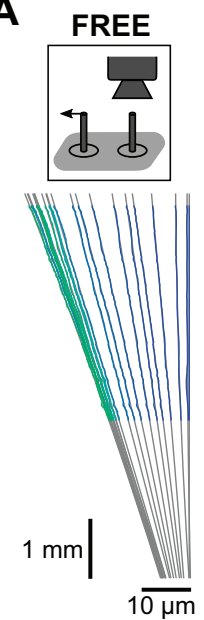

CONSTRAINED
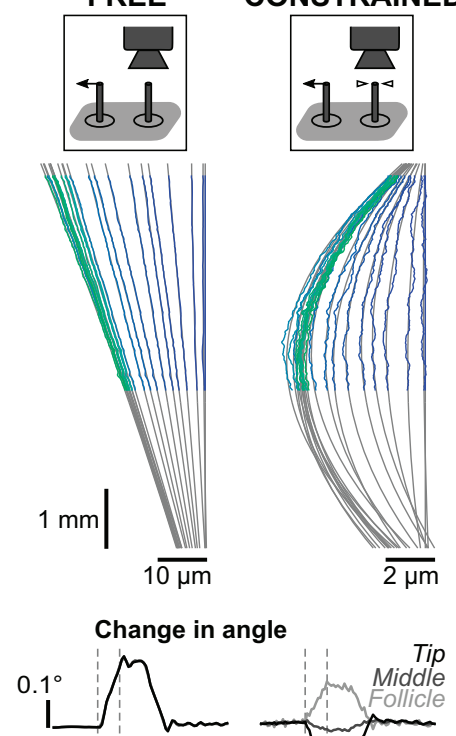

angle

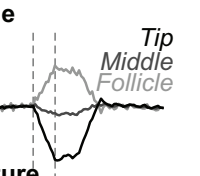

Change in curvature
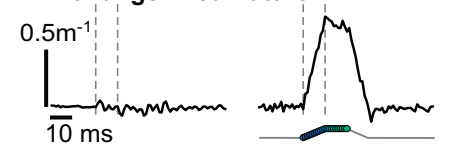

B

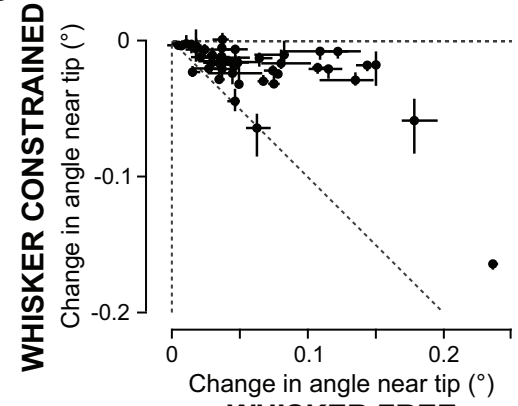

WHISKER FREE

C

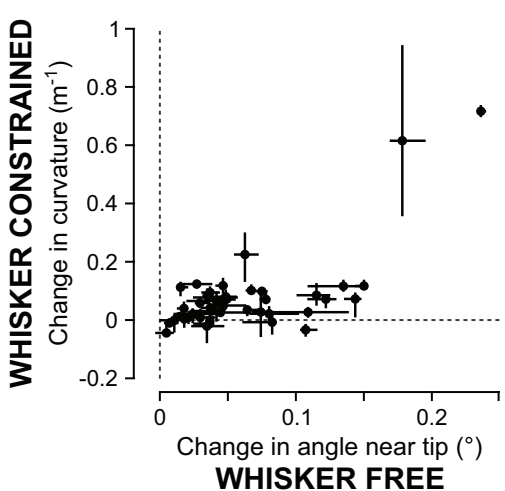

Fig. 4. Constraining the whisker tip results in a curvature change. A, top: deformation profiles (colored lines) and fits (gray lines) for a whisker either free (left) or constrained at its tip (right) while its neighbor is being deflected. A 10-point smoothing procedure was applied solely for visual display. Note that the horizontal scales are different in the 2 conditions. Bottom: time course of change in angle and change in curvature at 3 different levels along the shaft (tip, middle, and follicle, where "tip" indicates the point at which the stimulation contacts the whisker shaft) for the 2 conditions. The induced movement was a rigid rotation in the free condition and a bend of the whisker in the constrained condition. $B$ : change in angle near the tip of the whisker in the constrained condition vs. the free condition $(n=46$ whisker pairs; Spearman's coefficient $\left.\rho=-0.56, P=5.4 \times 10^{-5}\right)$. In $B$ and $C$, each point represents one combination of whiskers. Data points are the median value calculated over the 8 trials (4 rostral and 4 caudal, mirrored), and error bars indicate the 25 th and 75 th percentiles. $C$ : change in curvature in the constrained condition vs. change in angle in the free condition ( $n=46$ whisker pairs; Spearman's coefficient $\rho=0.47, P=0.001$ ). The very large error bar for one point comes from one case of different absolute values between rostral and caudal evoked movements. 
A

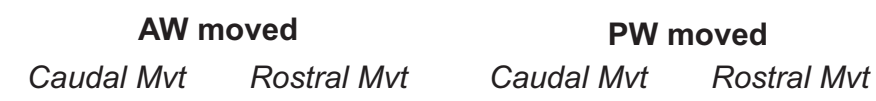

Fig. 5. Primary afferent neurons fire in response to their principal whisker (PW) and to a coupled neighbor whisker. A: action potential raster plots and peristimulus time histograms (1-ms bins) of the spiking activity of 3 example neurons, evoked by stimulation of their PW and an adjacent whisker (AW) for which mechanical coupling was revealed. Plots in two left and two right columns display responses to deflections of the AW and PW, respectively, whether it is the rostral or caudal whisker of the pair, for caudal and rostral deflections as indicated above the columns. The stimulation time course is shown below each histogram. For example neuron 1, spiking activity is shown for 2 different stimulation levels. For each neuron, the response to the $\mathrm{PW}$ in the $\mathrm{PW}$ preferred direction is indicated with an open background. The response to the AW in the AW preferred direction is indicated with a shaded background, highlighting the mechanical coupling effect at high stimulation levels. Parameters are neuron 1, low stimulation: $4^{\circ}, 800^{\circ} / \mathrm{s}, n=53$ trials, and high stimulation: $4^{\circ}, 1,330^{\circ} / \mathrm{s}, n=42$ trials; $n$ neuron $2: 3^{\circ}, 300^{\circ} / \mathrm{s}, n=60$ trials; neuron 3 , PW (D1): $4^{\circ}, 1,330^{\circ} / \mathrm{s}$; AW (delta): $\sim 11^{\circ}$, $\sim 3,800^{\circ} / \mathrm{s}, n=68$ trials. In this last case, the piezo tip for delta was advanced to $\sim 3 \mathrm{~mm}$ from follicle entry. $B$ : summary of mechanical coupling effects resulting in spiking activity. Each arrow indicates that spiking activity could be elicited in a neuron with its PW located at the end of the arrow while a neighboring AW, located at the start of the arrow, was deflected $(n=14$ neurons on 6 rats). Closed (or open) arrows indicate cases where the AW was a caudal (or rostral) neighbor. Darker whisker symbols indicate whiskers for which we obtained at least one primary afferent recording on which we were able to thoroughly test responses. Mvt, movement; pref. dir., preferred direction; sp., spike; stim., stimulation.

Example Neuron 1
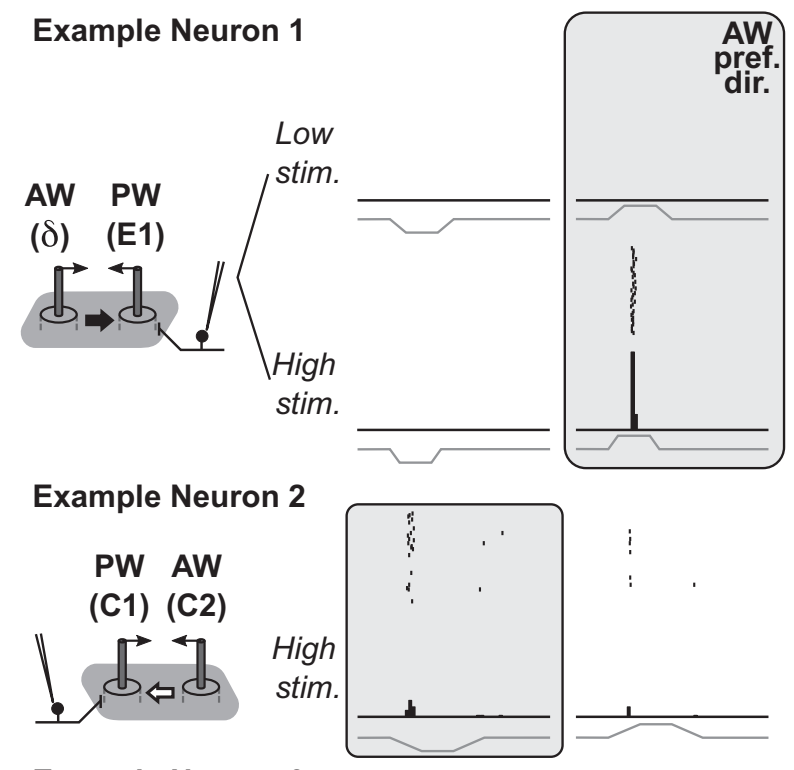

Example Neuron 3

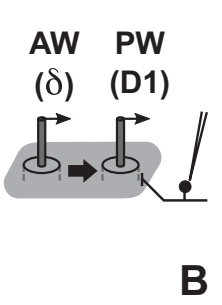

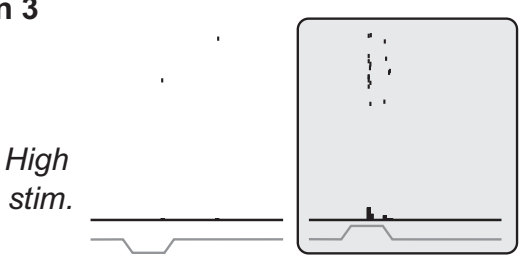

B
Arc $1 \quad$ Arc $2 \quad$ Arc $3 \quad$ Arc 4

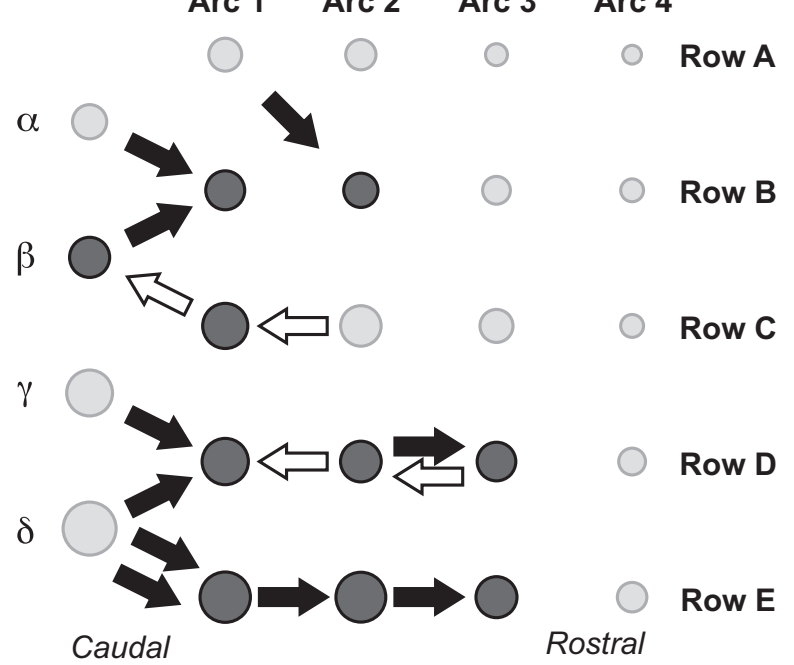

coupling effect. Interestingly, the direction of movement that had to be applied to the adjacent whisker to evoke spikes was opposite to the preferred direction for the principal whisker.

We report 14 cases of mechanical coupling leading to evoked spikes in TG neurons, of 14 TG recordings for which we were able to test responses to caudal and rostral neighboring whiskers at high deflection speeds (up to $4,000^{\circ} / \mathrm{s}$; see MATERIALS AND METHODS). On the summary map of Fig. $5 B$, each arrow indicates coupling from an adjacent whisker whose deflection evoked spikes in a TG neuron innervating a neigh- boring follicle. The functional response resulting from mechanical coupling was almost always observed for the stimulation of an immediately adjacent whisker in the same row, although it was always tested for all other 23 whiskers. We observed only one case of coupling across two different rows, from whisker A1 to whisker B2. Interestingly, most coupling effects (10/14; closed arrows in Fig. 5B) originated from the caudal adjacent whisker, in agreement with the larger mechanical coupling revealed in the videography experiments from immediately caudal whiskers (Figs. $2 B$ and $3 A$ ). The example 
neuron 2 of Fig. $5 A$ displays one of the four rostral interactions that we observed (open arrows in Fig. $5 B$ ). In this example, whisker $\mathrm{C} 2$ deflections elicited spikes in a C1-innervating neuron. Finally, preferred directions for the principal whisker and adjacent whiskers were usually opposite, except in two cases, one of which is shown in the bottom row of Fig. $5 \mathrm{~A}$ (example neuron 3).

\section{Adjacent Whisker Deflections Can Modify Responses of TG Neurons to Principal Whisker Deflections}

These recordings confirmed that deflecting an adjacent whisker could induce spiking activity in a TG neuron. However, it should be emphasized that this usually required strong stimulation pulses, from 330 to $\sim 4,000^{\circ} / \mathrm{s}$, thus above the stimulation thresholds observed for principal whisker stimulation in our sample (usually $100 \%$ s or less). Nonetheless, we reasoned that even subthreshold stimulation of an adjacent whisker could induce deformation of a follicle and modulate the firing properties of mechanoreceptors. We present one example cell suggesting that this subthreshold modulation can indeed occur. The middle row of Fig. $6 \mathrm{~A}$ displays the action potentials and average activity in time of a TG neuron for 36 rostral deflections of its principal whisker, D1, for two different speeds (left, $12.5 \%$; right, $20 \%$ s). During the same protocol, we also tested responses obtained when a deflection of whisker D2 was added, either in the caudal (Fig. 6A, top row) or in the rostral (bottom row) direction. In these randomly interleaved trials, D2 was deflected at a subthreshold stimulation level. We observed that the joint stimulation of D2 and D1 led to either more activity (Fig. 6A, top row, D2 caudally deflected) or less activity (bottom row, D2 rostrally deflected) than the single stimulation of D1. These effects were present for a range of stimulation speeds of the principal whisker and disappeared at very small speeds, when the principal whisker response itself occurred at a very long latency (Fig. 6B). Although we could not test this modulatory effect systematically, it suggests that neighboring whiskers have an ongoing influence on responses to the principal whisker through mechanical coupling.

\section{Mechanical Model of Two Neighboring Whiskers and Follicles}

Videography and electrophysiology experiments give us important but indirect clues about the mechanical interactions between follicles inside the skin. To estimate the transfer of mechanical forces from one follicle to a neighboring one and gain better understanding of its functional implications, we built a finite-element model of two whiskers and their follicles, linked by a layer of skin that takes into account superficial muscles (Fig. 7A). We used geometrical and mechanical parameter values in the ranges reported in the literature (see MATERIALS AND METHODS). The static deformations and forces resulting from the deflection of one whisker were calculated using SolidWorks Simulation. The model reproduced the expected induced movement of an adjacent whisker when one whisker is deflected at the tip (Fig. 7B, left, deflected whisker; middle, adjacent whisker). The change in angle, when the whisker was free in air, reached $0.2^{\circ}$ (20\% of the imposed deflection), in the same range as the experimental measurement shown in Fig. 1, and the external whisker shaft was straight (no curvature). When the whisker was constrained at the tip, it
A
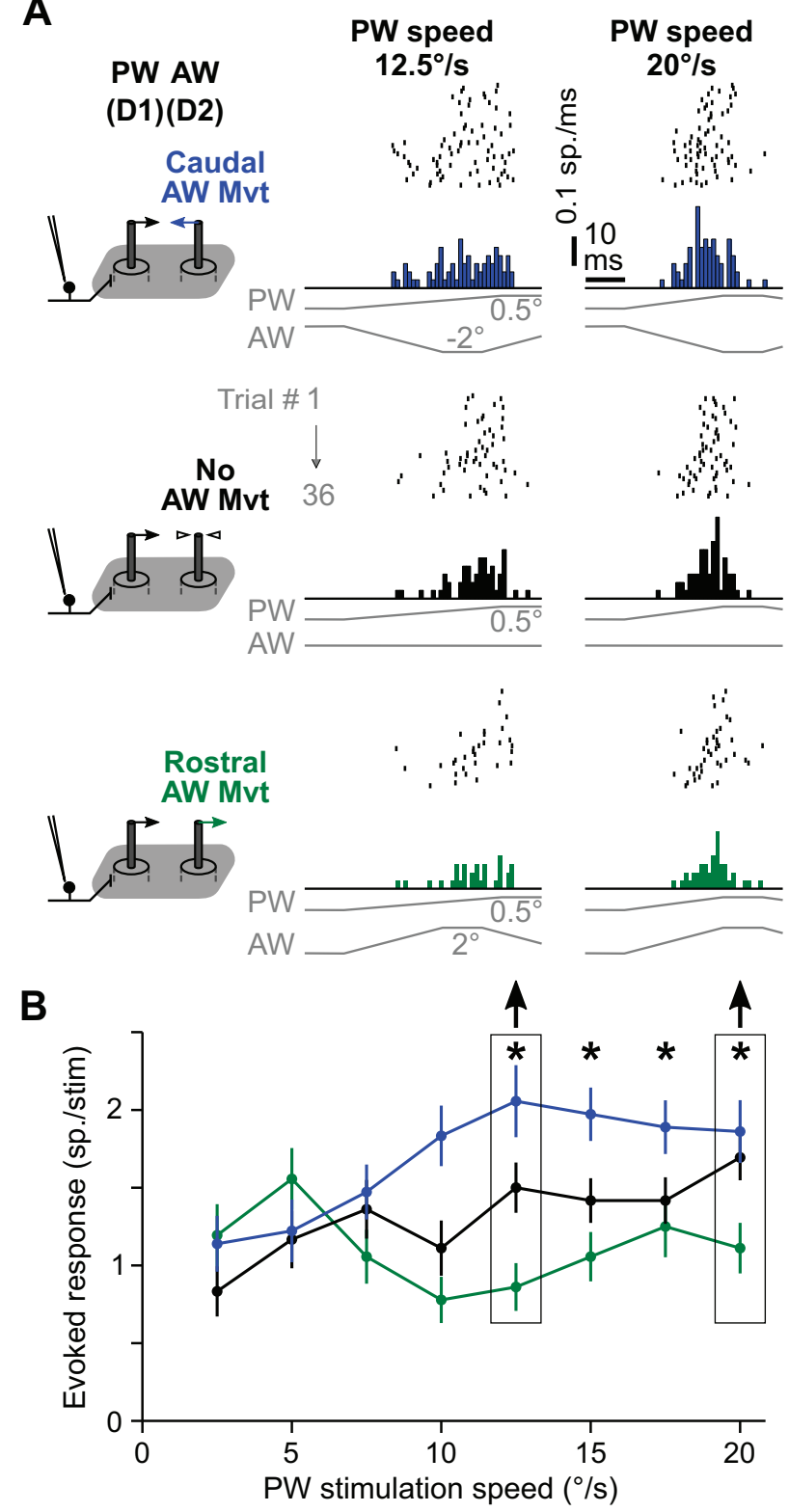

Fig. 6. Mechanical coupling between whiskers can modulate the response of a primary afferent neuron to its principal whisker (PW). A: raster plots and peristimulus time histograms of response of a neuron to rostral deflections of its PW at 2 different speed levels (left, $12.5 \%$ s; right, 20\%) while its adjacent whisker (AW) is either deflected caudally (top row), not deflected (middle row), or deflected rostrally (bottom row). B: evoked response (mean \pm SE in time window from start of stimulation to $10 \mathrm{~ms}$ after the end of the PW ramp) for 8 speed levels $\left(n=36\right.$ trials each). ${ }^{*} P \leq 0.01$, significant effect of AW direction (Welch's unpaired $t$ test).

showed changes in curvature along the whisker shaft (Fig. 7B, right), as was observed in the experimental data. The curvature reversed inside the follicle so that the whisker had an inverted $\mathrm{S}$ shape inside the follicle and protruded rostrally. When the whisker was free in air, there was only a $\mathrm{C}$-shaped bend inside the follicle.

We were particularly interested in the forces generated inside the follicles, which are the source of the input signals for downstream neural sensory processing. We extracted the contact pressure of the deflected and adjacent whiskers on their follicles, thus obtaining two-dimensional profiles of forces 
A

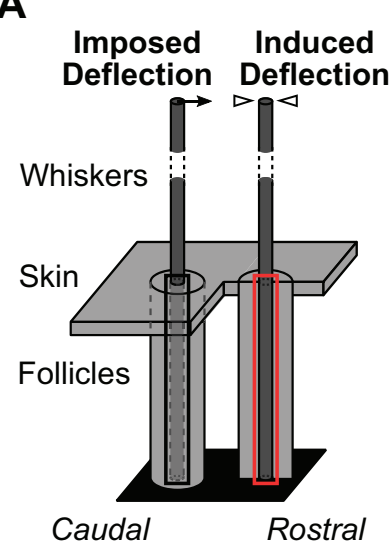

C

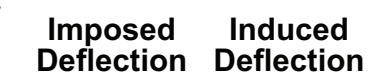

$B$

\section{Imposed Deflection Induced Deflection Induced Deflection Free \\ Constrained}

Displacement Curvature Displacement Curvature Disp
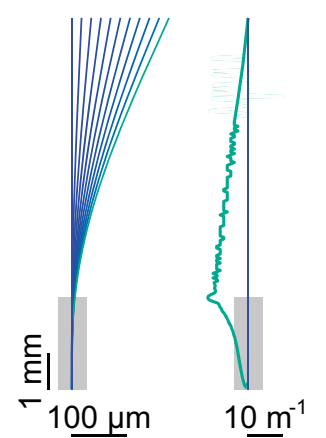
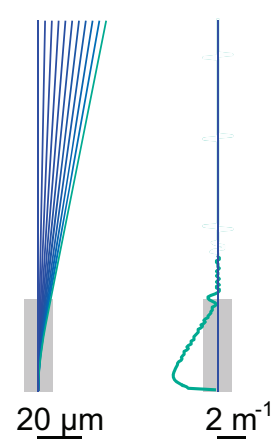

Displacement Curvature

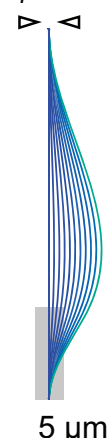

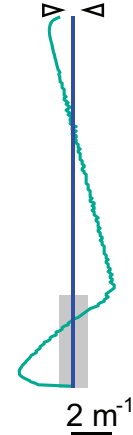

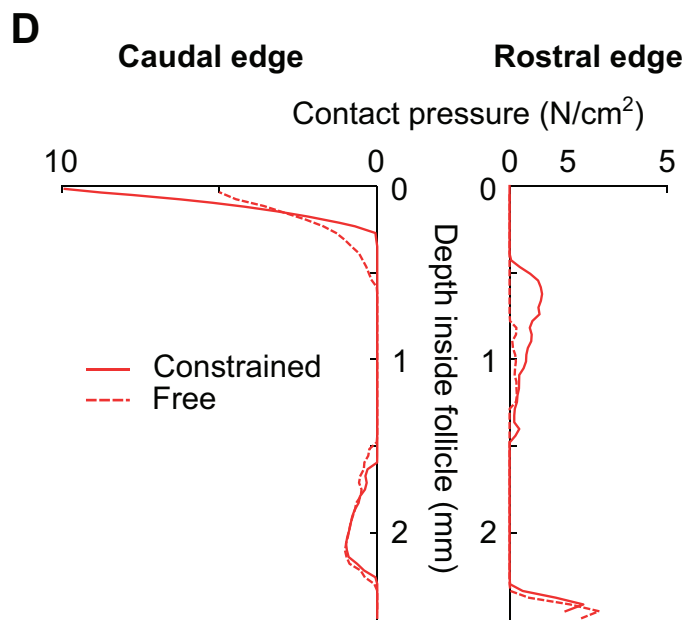

$\mathbf{F}$

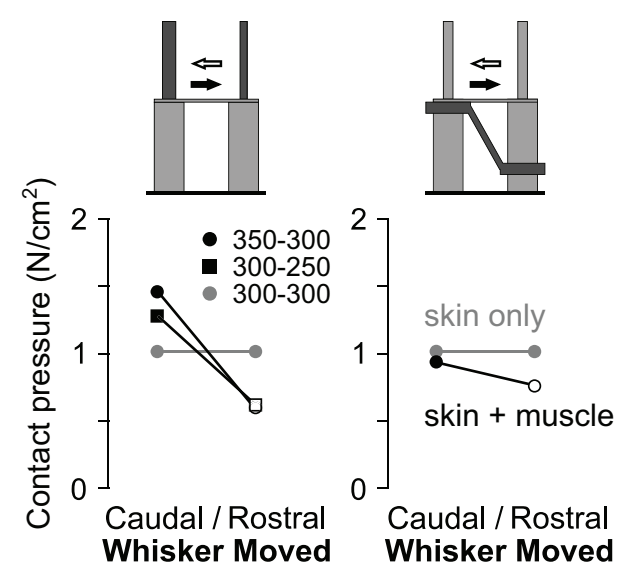

Fig. 7. A simple finite-element model predicts the location of mechanoreceptor activation due to mechanical coupling and the impact of whisker diameter. A: schematics of the model. Whiskers are represented as slender cylinders inside the follicles. The base of the whiskers and cylinders is fixed. The skin is modeled by a sheet embedding the top of the follicles. On this schematic, the front right quarter of the model has been removed to show the whisker-follicle arrangement. The left whisker is deflected by moving its tip while the right whisker is either left free or constrained. Caudal is arbitrarily set to the left. Note that some dimensions have been altered to better show the overall structure. $B$ : displacement and curvature along the 2 whiskers. The profiles are shown for 10 deflections of 0.1 to $1^{\circ}$, thus corresponding to experimental profiles of Figs. 1 and 4 . Negative curvature corresponds to a $\mathrm{C}$-shaped bend and positive curvature to its mirror image. $C$ : snapshot from SolidWorks Simulation of the contact pressure at the whisker-follicle interface, for the deflected whisker (left) and the neighbor whisker (right). The color scale was adjusted to best show the distribution of pressure; the overall maximum value was $30 \mathrm{~N} / \mathrm{cm}^{2}$. D: contact pressure on the caudal and rostral edges of the whisker-follicle interface for the induced deflection. Solid lines represent the constrained condition, and dashed lines represent the free condition. A 2-point running average was performed to attenuate mesh size artifacts. $E$ : same curves as in $D$ for the induced deflection in the constrained case, but with the diameter of the deflected whisker varying from 250 to $350 \mu \mathrm{m}$. Note that the contact pressure scale is different than in $D$. F , left: peak induced contact pressure in the upper middle zone for 3 different combinations of whisker diameters (in $\mu \mathrm{m}$ ). The thicker whisker was always caudal. Right: peak induced contact pressure in the upper middle zone for identical $300-\mu \mathrm{m}$ whiskers with or without the intrinsic muscle element added to the model. 
represented on two cylinders (Fig. $7 C$ ). For the whisker that was deflected at its tip in a rostral direction, these forces were distributed in several areas: mainly a rostral zone in the very top part and a large caudal zone in the upper middle (Fig. 7C). This distribution of forces matches the deformation of the whisker toward the front, i.e., the negative curvature in Fig. 7B, left. Functionally, the upper middle zone of the follicle is thought to contain the highest density of mechanoreceptors (Ebara et al. 2017). Our simulation results suggest that they would be activated because of the deflected whisker bending inside the follicle and pushing internally on that zone.

For the neighboring whisker, we further extracted the onedimensional profiles on the caudal and rostral lines along the cylinder modeling its follicle (Fig. 7D). The distribution of contact forces depended on whether the whisker shaft was let free in air or constrained at the tip. Specifically, when it was in the constrained condition, we obtained contact pressure areas mirroring those of the deflected whisker in the upper part of the follicle, with a reduced amplitude (Fig. 7, $C$ and D). Thus forces were present in a caudal zone at the top and a rostral zone in the upper middle part of the follicle. This distribution matches the S-shaped whisker bending revealed by the displacement and curvature profiles (Fig. $7 B$ ).

By contrast, when the nonmanipulated whisker was let free in air, the middle rostral zone of positive contact pressure largely disappeared (Fig. 7D). This corresponds to the whisker bending smoothly toward the front (Fig. 7B). The top caudal zone was still present, but contact forces were smaller. Overall, this result confirms that the free and constrained conditions indeed lead to different distributions of forces inside the follicle, and thus potentially to different ensembles of activated mechanoreceptors. In the following, we focus on the constrained condition and on contact pressure values in the upper middle zone of the follicle, given its importance in coding whisker deflections.

Note that if the imposed deflection is applied in the opposite direction, i.e., caudally, the displacement, curvature, and distribution of forces of the deflected and induced whisker-follicle ensembles are essentially symmetric to the rostral case. The mechanical coupling strength between the two whiskers is thus independent of the direction of stimulation, as in the videography experiments (Fig. 2A).

Using this simple model, we tested the causal link between whisker diameter and the amplitude of mechanical coupling. We increased or decreased the whisker diameter by $\sim 17 \%$ (50 $\mu \mathrm{m})$, which is in the range of whisker diameter differences between neighbors in a row of the whisker pad. When we modified the diameter of the deflected whisker while keeping the neighboring whisker diameter at $300 \mu \mathrm{m}$, the peak contact pressure inside the follicle varied by $40-45 \%$ (Fig. $7 E$ ). The curvature along the whisker changed little (350 $\mu \mathrm{m}: 7 \%$ decrease; $250 \mu \mathrm{m}$ : $15 \%$ increase). However, the whisker stiffness was almost doubled for the $350-\mu \mathrm{m}$ whisker, and conversely halved for the $250-\mu \mathrm{m}$ whisker. The bending moment and whisker-follicle forces were thus largely governed by the whisker diameter via the change in stiffness. This result confirms from a biomechanical point of view that the whisker diameter could indeed be a major factor in mechanical coupling effects.

We quantified the asymmetry created by these differences in diameter by the peak contact pressure in the upper middle zone
(Fig. 7F, left). We found that when the thicker whisker of an asymmetric pair was deflected, it induced a peak contact pressure in the neighboring follicle two times larger than when the thinner whisker was deflected. This ratio is in the range of what has been measured experimentally on induced deflections (Fig. $3 B$, mean change in angle $0.077^{\circ}$ vs. $0.042^{\circ}, n=14$ whisker pairs). Additionally, we observed in the model that the deflection of a whisker of 300- $\mu \mathrm{m}$ diameter had a stronger effect on a $250-\mu \mathrm{m}$ neighbor than on a 350- $\mu \mathrm{m}$ neighbor (contact pressure 1.26 vs. $0.60 \mathrm{~N} / \mathrm{cm} 2$; Fig. $7 F$, left, closed square vs. open circle), thus reproducing the asymmetric results of Fig. $3 C$ for a given deflected whisker. We conclude from these simulations that the distribution of mechanical coupling strength observed in the experiments, as well as the rostrocaudal asymmetry, can be fully explained by the gradient of whisker diameter.

Certainly, other elements of the model could be modified to study their impact on the mechanical coupling and its anisotropy. Increasing the follicle diameter tended to reduce coupling, probably because of increased mechanical absorption by the follicle. Increasing whisker spacing from 2 to $5 \mathrm{~mm}$ had very little effect ( $6 \%$ decrease in peak contact pressure).

Given that these geometrical parameters could not explain the mechanical coupling anisotropies, we then sought to test the impact of the different muscles of the whisker pad. Extrinsic muscles in the model were part of the skin volume. We found that modifying the skin thickness had little effect $(<3 \%$ for twice the thickness), even when a 2:1 thickness gradient was created along the rostrocaudal axis. On the other hand, doubling Young's modulus of the skin material increased the peak contact pressure by $60 \%$. Interestingly, it did not change the distribution of the contact forces inside the follicle. Indeed, this distribution is essentially governed by the boundary conditions at both ends of the whisker and can be changed by modifying those boundaries, as in the free vs. constrained conditions (Fig. 7, $B$ and $D$ ). Overall, these results from investigation of skin parameters suggest that a gradient of skin stiffness might contribute to the caudorostral gradient of mechanical coupling observed in the experiments.

Finally, we modeled the intrinsic muscle by a stiff oblique rod connecting two rings placed around the follicles (Fig. $7 F$, right). The presence of this asymmetric element created an asymmetry of coupling between the two whiskers while decreasing both values. Although the real intrinsic muscle is likely to be weaker than modeled here, this result suggests it could participate to the mechanical coupling asymmetry between whiskers.

From this simple model, we conclude that the gradient of mechanical coupling observed in the videography and electrophysiology experiments could be explained largely by the gradient in whisker diameter, with a possible contribution of the intrinsic muscles connecting the follicles.

\section{DISCUSSION}

In this study, we show that deflection of a single whisker on the rat snout is accompanied by measurable movements of neighboring whiskers. Moreover, if the deflection is sufficiently strong, it evokes spiking activity in primary afferent neurons innervating the follicles of neighboring whiskers. We use a simple mechanical model to show how transmission of 
mechanical forces through the skin is responsible for this cross-whisker interaction.

\section{Characteristics of Mechanical Coupling and Possible Underlying Mechanisms}

Both our videography and electrophysiology results emphasized intra-row interactions relative to intra-arc ones (Figs. $2 B$ and $5 B$ ). This could be due to the particular direction of our stimuli, along the rostrocaudal axis (see Methodological Considerations). This bias could also arise from the presence of intrinsic muscles between adjacent follicles in a row (Dörfl 1982; Haidarliu et al. 2010), responsible for pivoting the whisker around the follicle entry during whisking protraction. Recently, several laboratories reported the presence of additional oblique intrinsic muscles further connecting follicles along rows (Grant et al. 2013, 2017; Haidarliu et al. 2017). Together, these intrinsic muscles likely increase the stiffness of the skin particularly along the rostrocaudal direction, thus favoring the transmission of movements and forces along rows compared with arcs. Interestingly, intrinsic muscles can connect a straddler to both anterior follicles (Dörfl 1982), a pattern corresponding to the interaction of straddlers with both neighbors in our electrophysiological data (Fig. 5B).

A second striking result concerns the spatial distribution of mechanical coupling across the whisker pad. We found that effects were largely biased to the caudal half of the whisker pad, with an additional ventral emphasis especially in the electrophysiological data (Figs. $3 D$ and $5 B$ ). These results have led us to hypothesize that the size of the whisker, known to exhibit a strong caudoventral bias (Belli et al. 2017), could be an important factor governing the amplitude of mechanical coupling. In fact, many biomechanical studies assume the whisker diameter to be the only parameter distinguishing one whisker from another when describing the preneuronal transformation of contact events into forces at the follicle entry (Boubenec et al. 2012; Carvell and Simons 2017; Oladazimi et al. 2018; Quist and Hartmann 2012). Indeed, the hypothesis that mechanical coupling varies due to the gradient of the deflected whisker diameter seems the most parsimonious interpretation of our data. Interestingly, the dependence on the deflected whisker diameter was very strong in the biomechanical simulations, whereas the model was constructed and calibrated without this test in mind. Increasing the diameter of the whisker by $\sim 17 \%$, as is found between neighbors in a row, increases its stiffness by $85 \%$, according to the power law with exponent 4 applying to a cantilevered beam. An identical displacement at the tip thus requires a much larger bending moment and induces larger contact forces between the whisker and the follicle (Fig. 7E). The whisker diameter gradient could thus account for the overall spatial distribution of the crosswhisker coupling effect on the snout.

To definitively establish whisker diameter as a main factor in shaping the gradient of mechanical coupling on the whisker pad, several concerns will need to be addressed. First, the expected ventral bias was not clear in our videography data. Unfortunately, we could not test all combinations of deflected and imaged whiskers (see Methodological Considerations).

Second, the distribution of coupling effects revealed in the electrophysiology experiments necessarily includes a recording bias. In the TG, the number of neurons innervating large whisker follicles is higher than for small whiskers (Welker and Van der Loos 1986; Zucker and Welker 1969). There could also be a systematic bias in our electrode location in the ganglion, which is known to be loosely topographically organized (Leiser and Moxon 2006). Nonetheless, the strong caudoventral gradient suggests predominant mechanical coupling effects in that part of the pad. Note that we do not rule out the possibility that mechanical coupling could potentially influence the firing of any whisker-sensitive TG neuron, provided a neighbor whisker is deflected with sufficiently high magnitude.

In addition to the spatial gradient, for a given whisker combination, we found an asymmetry favoring mechanical coupling from the caudal to its immediately rostral neighbor, compared with the opposite sequence (Fig. 3B). We will consider several possible sources of this asymmetry. First, the systematic gradient in whisker diameter across the pad can suffice to explain a strong asymmetry in coupling inside a given whisker pair, as confirmed by the model simulations (Fig. 7, $E$ and $F$ ). This gradient can also explain that deflecting a fixed whisker affects differently its two neighbors (Fig. 3C), because the mechanical coupling strength is clearly dependent on the whisker diameter of the neighbor (Fig. $7 F$, left). Thus the rostral vs. caudal asymmetry observed in the experimental data can be fully explained by the gradient in whisker diameter across the pad.

Another factor that could play a role in the asymmetry of the mechanical coupling is the intrinsic muscle between follicles. As mentioned already, this muscle is attached to the superficial part of the caudal follicle and skin, and to the deep part of the rostral follicle. We have investigated the potential asymmetric mechanical effects resulting from this diagonal muscle by adding it to the biomechanical model as a rod connecting the follicles. We found that it could create an asymmetry favoring stronger coupling from the caudal whisker while at the same time reducing the overall values (Fig. $7 F$, right). Note that in the simulations, we chose to use the same material for the intrinsic muscle as for the skin and follicles, even though the real muscle is likely to be much less stiff than the tough, protective skin layer on the snout. The effect of the intrinsic muscle is thus probably largely overestimated in the model. Overall, we conclude that the intrinsic muscle could contribute to the asymmetry in cross-whisker effects but that it has probably much less influence than whisker diameter.

Other factors beyond whisker diameters and intrinsic muscles could contribute to the amplitude of mechanical coupling and to its asymmetry. For example, the size of follicle elements and surrounding muscles vary in a systematic way, correlated with the average whisker diameter (Haidarliu et al. 2010), and are likely to influence the transmission of forces from one whisker-follicle to another. The superficial extrinsic muscles maxillolabialis and nasolabialis probably stiffen the pad and could thus increase mechanical coupling and its asymmetry, in particular when the muscle tone is high, such as in the active exploring state. Moreover, because there are more fibers in the caudal section of the pad, the extrinsic muscles could contribute to the gradient that we observed. The nasolabialis fibers extending from the dorsocaudal region could particularly enhance mechanical coupling in that region compared with the ventrocaudal gradient expected from the gradient in whisker diameter. 
Our simulations suggested that the size of the follicles and the skin thickness were unlikely to explain the mechanical coupling distribution but that the stiffness of the skin layer could be important. This opens the possibility that the tone of the extrinsic muscles could have a significant impact. A more detailed model could be built to study specifically the effect of the different whisker pad elements. It could include variations in whisker taper and whisker low-density core (medulla), which recently were shown to vary across the pad, beyond the expected variations of length and diameter (Belli et al. 2017). Finally, in addition to these established gradients, an important source of variability could arise from the current phase of the whisker in the growth cycle, affecting directly its size (Ibrahim and Wright 1975).

\section{Distribution of Forces Activating Mechanoreceptors}

The biomechanical model was constructed to qualitatively bridge the gap between external whisker deformations and spiking activity in primary afferent neurons. We included only one element per whisker and follicle, embedded in a skin sheet. Minimal calibration was necessary to produce induced deformations compatible with experimental measures. With this simple model, the whisker shaft, when deflected, compresses the leading edge of the top of the follicle, as well as the trailing edge at a deeper location because of internal bending. This agrees with a recent ex vivo study (Whiteley et al. 2015) describing a distribution of strain along the depth of the follicle in which compression and dilation zones alternate. When the neighboring whisker is constrained at its tip, induced contact forces on its follicle adopt a mirror configuration with a smaller amplitude (Fig. 7C). As a consequence, a mechanoreceptor located in a zone of compression for an imposed deflection of its principal whisker will be best stimulated if the adjacent whisker is deflected in the opposite direction. Indeed, in most $(12 / 14)$ TG neurons, we observed that the preferred direction of deflection for the adjacent whisker was opposite to the preferred direction for the principal whisker (Fig. 5).

The density of mechanoreceptors is highest in the top half of the follicle, and more specifically in the ring sinus region (Ebara et al. 2017). In a recent study combining anatomical and functional characterization, the most numerous and most responsive TG neurons were found to be those terminating with clublike endings in the ring sinus region. These terminals are particularly suited to encode a specific direction of movement (Tonomura et al. 2015). This class of mechanoreceptors has previously been underestimated because of their tiny axonal endings, easily mistaken as cut axons. Interestingly, our model does predict contact forces in this region induced by deflection of an adjacent whisker, particularly when the principal whisker is constrained (Fig. 7).

In this respect, we have systematically explored two different conditions in the experiments and the model: the free and constrained conditions. It is interesting to note that constraining the neighboring whisker led to increased curvature changes along the shaft and, at the same time, to increased responses in the putative mechanoreceptors of the associated follicle. This is in line with the idea that the rotational moment at the base of the whisker, known to be proportional to the curvature, is indeed what is encoded by mechanoreceptors, in both the passive and active states (Campagner et al. 2016; Quist and
Hartmann 2012). The model simulations, by illustrating the distribution of forces inside the follicle, offer a mechanistic explanation. When the whisker is constrained, a large contact pressure zone is present in the ring sinus region, compared with the free condition (Fig. 7C). Thus the model confirms that the external forces on the whisker produce a bending moment along the shaft that causes pressure forces in a specific localized zone of the follicle. Mechanoreceptors terminating in this zone are activated, encoding directional and amplitude information about the deflection event.

\section{Functional Relevance}

Our results imply that the skin tissue and muscles making up the whisker pad transmit forces between follicles and that this additional force field can, depending on its direction, either counteract or augment the ongoing forces due to external events on the whisker shaft. It brings forward a theory that has already been proposed, namely, that the whisker system, despite its discreteness, could function as a continuous sensory organ just as the skin does (Simons 1995).

From a practical point of view, our results imply that there can be no pure single-whisker movement, because the biomechanical forces move all elements of the pad (muscles, follicles, whiskers) in an automatic fashion. These induced deformations could reach up to $10-20 \%$ in our experimental conditions. Their impact on sensory processing should be considered. It has been shown that the most sensitive primary afferents respond to extremely small deflections of amplitude less than $0.01^{\circ}$, and one-third of all afferents have a velocity threshold below 3\% (Gibson and Welker 1983). For very small single-whisker deflections, only a handful of TG neurons spike action potentials, and all these neurons are likely to innervate the deflected whisker, conforming to the labeled line hypothesis. When deflection parameters increase, more and more mechanoreceptors are activated, including some with low thresholds located in surrounding follicles. Upstream, we thus expect some amount of divergence of the sensory signal from a single whisker to TG neurons innervating other whiskers, and further, to surrounding barrelettes. This divergence will of course depend on the particular whisker considered, and we propose that the whisker diameter is an important parameter of the extent of this spatial spread.

During stimulation of several whiskers overlapping in time, our results suggest that modulation of responses to the principal whisker by the simultaneous deflection of surrounding whiskers is also already present at the TG level. The example neuron of Fig. 6 shows that even for very low deflection values, mechanical coupling can modify responses to external events occurring on the principal whisker. As confirmed by the model, even low deflections modulate the distribution of forces present in neighboring follicles. When two deflections occur at the same time, forces inside the follicles will be enhanced for opposite directions of movement or, on the contrary, attenuated. Functionally, these ongoing mechanical effects could for example emphasize detection of surfaces tending to bring whiskers together, such as corners.

In our view, this peripheral cross-whisker interaction can be thought of as a first nonlinearity in the information processing pathway, before the other known nonlinearities at the trigeminal nuclei, thalamic, and cortical levels. Several laboratories, 
including our own, have reported that cortical neurons are able to extract multiwhisker features of tactile scenes (review in Estebanez et al. 2018), suggesting that cortical neuronal tuning could underlie the ability of animals to identify relevant perceptual features. The existence of multiwhisker interactions within the whisker pad does not contradict these findings. Rather, it confirms that multiwhisker integration starts already before the cortex. In other words, we need to be careful about claiming that nonlinearities observed in the cortex are not already present at a subcortical or even peripheral level. Such mechanisms have been known to exist for a long time in the trigeminal nuclei (Minnery and Simons 2003; Timofeeva et al. 2004) and also have been described in the thalamus, including the extraction of high-order features such as global apparent motion (Ego-Stengel et al. 2012). In this last study, multiwhisker selectivity was shown to be present in the thalamus, but to a lesser extent than in the cortex, and to be amplified at the cortical level. Our current view on tactile processing mechanisms is that intracortical circuitry builds an additional layer of computation that uses the results of nonlinearities in the previous stages of the system to transform the tactile signals further. Future experiments should help to understand the precise role of each of these stages.

\section{Methodological Considerations}

Following the description of a single case of cross-whisker spiking response in a TG neuron (Simons 1985), this is the first study directly investigating mechanical coupling between whiskers and its consequences on neuronal encoding of tactile information. In most laboratories, including our own, only relatively low-amplitude, low-speed stimuli are routinely implemented. Indeed, a known limitation of piezoelectric stimulators, widely used in the field, is that high-velocity stimuli quickly produce ringing (Jacob et al. 2010), thereby constraining their useful range. In the summary drawn by Ritt and collaborators (2008), they concluded that the highest speed explored in electrophysiological studies across laboratories was $2,500^{\circ} \mathrm{s}$, and the highest amplitude of deflection was $3^{\circ}$. In the present study, we raised the deflection amplitude to $3^{\circ}$ or more, and the speed up to $4,000^{\circ}$ s, to reveal direct mechanical cross-whisker effects on neurons. These parameters could only be achieved by placing the stimulator close to the whisker base, a procedure performed very carefully under the microscope to touch neither the fur nor a neighboring whisker or stimulator.

The use of high-resolution videography allowed us to track the profile of whiskers with extreme precision, below $1 \mu \mathrm{m}$ and at $1 \mathrm{kHz}$, using minimal image processing. We quantified mechanical coupling effects in a systematic way by measuring the deformation profile of whiskers. We only imaged whiskers for which the shaft was relatively horizontal, thus in focus along its length, and unobstructed by bulging of the pad or by excessive fur. Future experiments could take advantage of new cameras that are more compact and easier to position with different angles around the animal, potentially allowing tracking of all macrovibrissae.

The experiments were performed on an anesthetized preparation to ensure stable conditions and full control of the stimulus. Assessing the magnitude of cross-whisker coupling in awake behaving animals will be particularly challenging. Animals will have to be trained, for example, by head fixation, to enable high-resolution imaging of their whiskers. Importantly, the awake preparation will introduce multiple factors that can influence the state of the follicles and that will require monitoring. The baseline tonus of skin muscles involved in the whisker array positioning is likely to be larger in the awake animal, possibly transmitting mechanical energy more efficiently across follicles. By using anesthesia, we may in fact have underestimated the coupling effect. Electromyographic recordings have further shown that the pad muscles are tightly regulated by a feedback loop triggered by whisker contact (Bellavance et al. 2017; Nguyen and Kleinfeld 2005). Accompanying changes in the pressure inside the follicle blood sinus could affect the receptors dynamic range of encoding. The magnitude of cross-whisker coupling is thus likely to vary continuously during the awake state, even in a passive condition.

When animals explore their environment, they actively move their whiskers in a coordinated way, including whisking but also asymmetric behaviors (Grant et al. 2009; Sofroniew and Svoboda 2015). The activation of the pad musculature controls rostrocaudal translation of follicles, along with the protraction of individual whiskers and more subtle deformations (pad bulging, whisker torsion, etc.). In our study, we have purposefully avoided these internally generated movements and focused on mechanical coupling effects at rest, extracting whisker profile deviations from a stable baseline. In an active animal, evaluating the impact of cross-whisker effects will require an analysis that can disentangle the movements due to active behavior from the movements due to skin coupling. We hypothesize that the passive mechanical coupling effects that we have described add to the underlying global movements of the different structures of the whisker pad. Thus an external touch on one whisker will modify the trajectory of that whisker but also that of neighboring whiskers relative to what it would have been without that touch. How this superposition of internally and externally generated deformations of follicles and whiskers translates into patterns of mechanoreceptor activation will have to be investigated in future studies.

\section{ACKNOWLEDGMENTS}

We thank Camila Pulido and David Davila for contributions to early whisker tracking and electrophysiology experiments. We are grateful to the whole Shulz laboratory for advice throughout the project, to Evan Harrell for comments on the manuscript, and to Yannick Passarelli for help finalizing the submission. We thank Guillaume Hucher for measuring whisker parameters and for trigeminal ganglion histology, and Aurélie Daret for help with animal experiments and general laboratory managing.

Present address of Y. Boubenec: Laboratoire des Systèmes Perceptifs, Département d'Etudes Cognitives, École Normale Supérieure, 29 rue d'Ulm, 75005 Paris, France.

\section{GRANTS}

This work was supported by International Human Frontier Science Program Organization Grant CDA 00044-2010, Agence Nationale de la Recherche (ANR) Grant NeuroWhisk ANR-14-CE24-0019-01, Equipe Fondation de la Recherche Médicale Grant DEQ20170336761, Paris-Saclay University (Lidex iCODE), and IDEX NeuroSaclay Grant ANR-11-IDEX-0003-02.

\section{DISCLOSURES}

No conflicts of interest, financial or otherwise, are declared by the authors. 


\section{AUTHOR CONTRIBUTIONS}

V.E.-S. and D.E.S. conceived and designed research; V.E.-S., A.A., M.L., and Y.B. performed experiments; V.E.-S., A.A., M.L., H.L., and Y.B. analyzed data; V.E.-S. interpreted results of experiments; V.E.-S. and H.L. prepared figures; V.E.-S. drafted manuscript; A.A., M.L., Y.B., and D.E.S. approved final version of manuscript; H.L. and D.E.S. edited and revised manuscript.

\section{REFERENCES}

Arnold PB, Li CX, Waters RS. Thalamocortical arbors extend beyond single cortical barrels: an in vivo intracellular tracing study in rat. Exp Brain Res 136: 152-168, 2001. doi:10.1007/s002210000570.

Axelrad H, Verley R, Farkas E. Responses evoked in mouse and rat SI cortex by vibrissa stimulation. Neurosci Lett 3: 265-274, 1976. doi:10.1016/03043940(76)90053-7.

Bagdasarian K, Szwed M, Knutsen PM, Deutsch D, Derdikman D, Pietr M, Simony E, Ahissar E. Pre-neuronal morphological processing of object location by individual whiskers. Nat Neurosci 16: 622-631, 2013. doi:10. 1038/nn.3378.

Bellavance MA, Takatoh J, Lu J, Demers M, Kleinfeld D, Wang F, Deschênes M. Parallel inhibitory and excitatory trigemino-facial feedback circuitry for reflexive vibrissa movement. Neuron 95: 673-682.e4, 2017. [Erratum in Neuron 95: 722-723, 2017.] doi:10.1016/j.neuron.2017.06.045.

Belli HM, Yang AE, Bresee CS, Hartmann MJ. Variations in vibrissal geometry across the rat mystacial pad: base diameter, medulla, and taper. $J$ Neurophysiol 117: 1807-1820, 2017. doi:10.1152/jn.00054.2016.

Bernardo KL, McCasland JS, Woolsey TA. Local axonal trajectories in mouse barrel cortex. Exp Brain Res 82: 247-253, 1990. doi:10.1007/ BF00231244.

Boubenec Y, Claverie LN, Shulz DE, Debrégeas G. An amplitude modulation/demodulation scheme for whisker-based texture perception. $J$ Neurosci 34: 10832-10843, 2014. doi:10.1523/JNEUROSCI.0534-14.2014.

Boubenec Y, Shulz DE, Debrégeas G. Whisker encoding of mechanical events during active tactile exploration. Front Behav Neurosci 6: 74, 2012. doi:10.3389/fnbeh.2012.00074.

Campagner D, Evans MH, Bale MR, Erskine A, Petersen RS. Prediction of primary somatosensory neuron activity during active tactile exploration. eLife 5: e10696, 2016. doi:10.7554/eLife.10696.

Carl K, Hild W, Mämpel J, Schilling C, Uhlig R, Witte H. Characterization of statical properties of rat's whisker system. IEEE Sens J 12: 340-349, 2012. doi:10.1109/JSEN.2011.2114341

Carvell GE, Simons DJ. Task- and subject-related differences in sensorimotor behavior during active touch. Somatosens Mot Res 12: 1-9, 1995. doi:10. 3109/08990229509063138.

Carvell GE, Simons DJ. Effect of whisker geometry on contact force produced by vibrissae moving at different velocities. J Neurophysiol 118: 1637-1649, 2017. doi:10.1152/jn.00046.2017.

Deschênes M, Urbain N. Vibrissal afferents from trigeminus to cortices. Scholarpedia 4: 7454, 2009. doi:10.4249/scholarpedia.7454.

Dörfl J. The musculature of the mystacial vibrissae of the white mouse. J Anat 135: 147-154, 1982.

Dykes RW. Afferent fibers from mystacial vibrissae of cats and seals. $J$ Neurophysiol 38: 650-662, 1975. doi:10.1152/jn.1975.38.3.650.

Ebara S, Furuta T, Kumamoto K. Vibrissal mechanoreceptors. Scholarpedia 12: 32372, 2017. doi: $10.4249 /$ scholarpedia. 32372.

Ebara S, Kumamoto K, Matsuura T, Mazurkiewicz JE, Rice FL. Similarities and differences in the innervation of mystacial vibrissal follicle-sinus complexes in the rat and cat: a confocal microscopic study. J Comp Neurol 449: 103-119, 2002. doi:10.1002/cne.10277.

Ego-Stengel V, Le Cam J, Shulz DE. Coding of apparent motion in the thalamic nucleus of the rat vibrissal somatosensory system. J Neurosci 32: 3339-3351, 2012. doi:10.1523/JNEUROSCI.3890-11.2012.

Estebanez L, Férézou I, Ego-Stengel V, Shulz DE. Representation of tactile scenes in the rodent barrel cortex. Neuroscience 368: 81-94, 2018. doi:10. 1016/j.neuroscience.2017.08.039.

Gibson JM, Welker WI. Quantitative studies of stimulus coding in first-order vibrissa afferents of rats. 1. Receptive field properties and threshold distributions. Somatosens Res 1: 51-67, 1983. doi:10.3109/07367228309144540.

Gottschaldt KM, Iggo A, Young DW. Functional characteristics of mechanoreceptors in sinus hair follicles of the cat. $J$ Physiol 235: 287-315, 1973. doi:10.1113/jphysiol.1973.sp010388.

Grant RA, Delaunay MG, Haidarliu S. Mystacial whisker layout and musculature in the guinea pig (Cavia porcellus): a social, diurnal mammal. Anat Rec (Hoboken) 300: 527-536, 2017. doi:10.1002/ar.23504.
Grant RA, Haidarliu S, Kennerley NJ, Prescott TJ. The evolution of active vibrissal sensing in mammals: evidence from vibrissal musculature and function in the marsupial opossum Monodelphis domestica. J Exp Biol 216: 3483-3494, 2013. doi:10.1242/jeb.087452.

Grant RA, Mitchinson B, Fox CW, Prescott TJ. Active touch sensing in the rat: anticipatory and regulatory control of whisker movements during surface exploration. J Neurophysiol 101: 862-874, 2009. doi:10.1152/jn. 90783.2008.

Haidarliu S. An anatomically adapted, injury-free headholder for guinea pigs. Physiol Behav 60: 111-114, 1996. doi:10.1016/0031-9384(95)02257-0.

Haidarliu S, Bagdasarian K, Shinde N, Ahissar E. Muscular basis of whisker torsion in mice and rats. Anat Rec (Hoboken) 300: 1643-1653, 2017. doi:10.1002/ar.23623.

Haidarliu S, Simony E, Golomb D, Ahissar E. Muscle architecture in the mystacial pad of the rat. Anat Rec (Hoboken) 293: 1192-1206, 2010. doi:10.1002/ar.21156

Hartmann MJ, Johnson NJ, Towal RB, Assad C. Mechanical characteristics of rat vibrissae: resonant frequencies and damping in isolated whiskers and in the awake behaving animal. J Neurosci 23: 6510-6519, 2003. doi:10. 1523/JNEUROSCI.23-16-06510.2003.

Hobbs JA, Towal RB, Hartmann MJ. Spatiotemporal patterns of contact across the rat vibrissal array during exploratory behavior. Front Behav Neurosci 9: 356, 2016. doi:10.3389/fnbeh.2015.00356.

Ibrahim L, Wright EA. The growth of rats and mice vibrissae under normal and some abnormal conditions. J Embryol Exp Morphol 33: 831-844, 1975.

Jacob V, Estebanez L, Le Cam J, Tiercelin JY, Parra P, Parésys G, Shulz DE. The Matrix: a new tool for probing the whisker-to-barrel system with natural stimuli. J Neurosci Methods 189: 65-74, 2010. doi:10.1016/j. jneumeth.2010.03.020.

Jacquin MF, Chiaia NL, Haring JH, Rhoades RW. Intersubnuclear connections within the rat trigeminal brainstem complex. Somatosens Mot Res 7: 399-420, 1990. doi:10.3109/08990229009144716.

Kim JN, Koh KS, Lee E, Park SC, Song WC. The morphology of the rat vibrissal follicle-sinus complex revealed by three-dimensional computeraided reconstruction. Cells Tissues Organs 193: 207-214, 2011. doi:10. $1159 / 000319394$

Knutsen PM, Pietr M, Ahissar E. Haptic object localization in the vibrissal system: behavior and performance. J Neurosci 26: 8451-8464, 2006. doi:10.1523/JNEUROSCI.1516-06.2006.

Krupa DJ, Matell MS, Brisben AJ, Oliveira LM, Nicolelis MA. Behavioral properties of the trigeminal somatosensory system in rats performing whisker-dependent tactile discriminations. J Neurosci 21: 5752-5763, 2001. doi:10.1523/JNEUROSCI.21-15-05752.2001.

Lavallée P, Deschênes M. Dendroarchitecture and lateral inhibition in thalamic barreloids. J Neurosci 24: 6098-6105, 2004. doi:10.1523/ JNEUROSCI.0973-04.2004.

Leiser SC, Moxon KA. Relationship between physiological response type (RA and SA) and vibrissal receptive field of neurons within the rat trigeminal ganglion. J Neurophysiol 95: 3129-3145, 2006. doi:10.1152/jn.00157. 2005.

Ma PM, Woolsey TA. Cytoarchitectonic correlates of the vibrissae in the medullary trigeminal complex of the mouse. Brain Res 306: 374-379, 1984. doi:10.1016/0006-8993(84)90390-1.

Minnery BS, Simons DJ. Response properties of whisker-associated trigeminothalamic neurons in rat nucleus principalis. J Neurophysiol 89: 40-56, 2003. doi:10.1152/jn.00272.2002.

Narayanan RT, Egger R, Johnson AS, Mansvelder HD, Sakmann B, de Kock CP, Oberlaender M. Beyond columnar organization: cell type- and target layer-specific principles of horizontal axon projection patterns in rat vibrissal cortex. Cereb Cortex 25: 4450-4468, 2015. doi:10.1093/cercor/ bhv053.

Neimark MA, Andermann ML, Hopfield JJ, Moore CI. Vibrissa resonance as a transduction mechanism for tactile encoding. J Neurosci 23: 64996509, 2003. doi:10.1523/JNEUROSCI.23-16-06499.2003.

Nguyen QT, Kleinfeld D. Positive feedback in a brainstem tactile sensorimotor loop. Neuron 45: 447-457, 2005. doi:10.1016/j.neuron.2004.12.042.

Oladazimi M, Brendel W, Schwarz C. Biomechanical texture coding in rat whiskers. Sci Rep 8: 11139, 2018. doi:10.1038/s41598-018-29225-9.

Quist BW, Hartmann MJ. Mechanical signals at the base of a rat vibrissa: the effect of intrinsic vibrissa curvature and implications for tactile exploration. J Neurophysiol 107: 2298-2312, 2012. doi:10.1152/jn.00372.2011.

Ritt JT, Andermann ML, Moore CI. Embodied information processing: vibrissa mechanics and texture features shape micromotions in actively sensing rats. Neuron 57: 599-613, 2018. doi:10.1016/j.neuron.2007.12.024. 
Schneider JS, Denaro FJ, Olazabal UE, Leard HO. Stereotaxic atlas of the trigeminal ganglion in rat, cat, and monkey. Brain Res Bull 7: 93-95, 1981. doi:10.1016/0361-9230(81)90103-9.

Sherman D, Oram T, Harel D, Ahissar E. Attention robustly gates a closed-loop touch reflex. Curr Biol 27: 1836-1843.e7, 2017. doi:10.1016/ j.cub.2017.05.058.

Simons DJ. Response properties of vibrissa units in rat SI somatosensory neocortex. J Neurophysiol 41: 798-820, 1978. doi:10.1152/jn.1978.41.3.798.

Simons DJ. Temporal and spatial integration in the rat SI vibrissa cortex. $J$ Neurophysiol 54: 615-635, 1985. doi:10.1152/jn.1985.54.3.615.

Simons DJ. Neuronal integration in the somatosensory whisker/barrel cortex. In: The Barrel Cortex of Rodents. Cerebral Cortex, edited by Jones EG, Diamond IT. Boston, MA: Springer, 1995, vol. 11, p. 263-297. doi:10.1007/ 978-1-4757-9616-2_6.

Sofroniew NJ, Svoboda K. Whisking. Curr Biol 25: R137-R140, 2015. doi:10.1016/j.cub.2015.01.008.

Solomon JH, Hartmann MJ. Biomechanics: robotic whiskers used to sense features. Nature 443: 525, 2006. doi:10.1038/443525a.

Timofeeva E, Lavallée P, Arsenault D, Deschênes M. Synthesis of multiwhisker-receptive fields in subcortical stations of the vibrissa system. $J$ Neurophysiol 91: 1510-1515, 2004. doi:10.1152/jn.01109.2003.

Tonomura S, Ebara S, Bagdasarian K, Uta D, Ahissar E, Meir I, Lampl I, Kuroda D, Furuta T, Furue H, Kumamoto K. Structure-function correlations of rat trigeminal primary neurons: Emphasis on club-like endings, a vibrissal mechanoreceptor. Proc Jpn Acad Ser B Phys Biol Sci 91: 560-576, 2015. doi:10.2183/pjab.91.560.
Van Der Loos H. Barreloids in mouse somatosensory thalamus. Neurosci Lett 2: 1-6, 1976. doi:10.1016/0304-3940(76)90036-7.

Vincent S. The tactile hair of the white rat. J Comp Neurol 23: 1-36, 1913. doi:10.1002/cne.900230101

Voges D, Carl K, Klauer GJ, Uhlig R, Schilling C, Behn C, Witte H. Structural characterization of the whisker system of the rat. IEEE Sens J 12: 332-339, 2012. doi:10.1109/JSEN.2011.2161464.

Voisin DL, Doméjean-Orliaguet S, Chalus M, Dallel R, Woda A. Ascending connections from the caudal part to the oral part of the spinal trigeminal nucleus in the rat. Neuroscience 109: 183-193, 2002. doi:10.1016/S03064522(01)00456-0.

Welker E, Van der Loos H. Quantitative correlation between barrel-field size and the sensory innervation of the whiskerpad: a comparative study in six strains of mice bred for different patterns of mystacial vibrissae. $J$ Neurosci 6: 3355-3373, 1986. doi:10.1523/JNEUROSCI.06-11-03355.1986.

Whiteley SJ, Knutsen PM, Matthews DW, Kleinfeld D. Deflection of a vibrissa leads to a gradient of strain across mechanoreceptors in a mystacial follicle. J Neurophysiol 114: 138-145, 2015. doi:10.1152/jn.00179.2015.

Woolsey TA, Van der Loos H. The structural organization of layer IV in the somatosensory region (SI) of mouse cerebral cortex. The description of a cortical field composed of discrete cytoarchitectonic units. Brain Res 17: 205-242, 1970. doi:10.1016/0006-8993(70)90079-X.

Zucker E, Welker WI. Coding of somatic sensory input by vibrissae neurons in the rat's trigeminal ganglion. Brain Res 12: 138-156, 1969. doi:10.1016/ 0006-8993(69)90061-4.

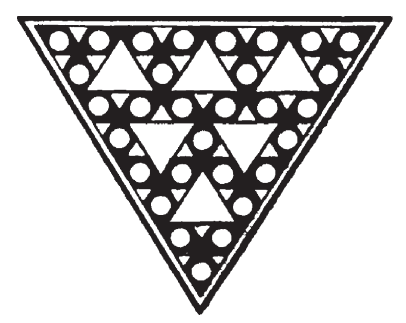

